BULL. AUSTRAL. MATH. SOC.

VOL. $13(1975), 169-210$.

\title{
Fuzzy machines in a category
}

\section{Michael A. Arbib and Ernest G. Manes}

\begin{abstract}
"Fuzzy theories" and "distributive laws" are used to define
"fuzzy systems" in an arbitrary category. The resulting minimal realization theory provides new insights even in classical cases (so that, for non-deterministic sequential machines, the minimal realization problem is reformulated in terms of the structure of join-irreducibles in finite lattices). The definition of "fuzzy theory" is of independent interest and meshes well with philosophical aspects of fuzzy set theory.
\end{abstract}

\section{Introduction}

Whereas an ordinary sequential machine has dynamics (state-transition function)

$$
Q \times X_{0} \rightarrow Q
$$

where $Q$ is the set of states and $X_{0}$ is the set of inputs; a nondeterministic sequential machine has dynamics

$$
\delta: Q \times X_{0} \rightarrow 2^{Q},
$$

where we interpret $\delta(q, x) \subset Q$ as the set of possible successors to $q$ when acted upon by input $x$. Again, a stochastic sequential machine (of a restricted type) has dynamics

$$
Q \times X_{0} \rightarrow Q P
$$

where $Q P$ is the set of probability distributions on $Q$; while

Received 21 May 1975. This research was supported by the National Science Foundation of the USA. This paper has been referred to elsewhere under the title "Kleisli machines". 
Schützenberger [17] studies what we call semiring automata with dynamics

$$
Q \times X_{0} \rightarrow R^{(Q)}=\{f: Q \rightarrow R \mid \operatorname{supp}(f) \text { is finite }\}
$$

for some fixed semiring ${ }^{1} R$.

In previous papers [1,4], we saw that the formation of $Q \times X_{0}$ from $Q$ could be generalized by considering any category $K$ and any functor $X: K \rightarrow K$; we formed a category $\operatorname{Dyn}(X)$, with objects $K$-morphisms $\delta: Q X \rightarrow Q$, while a morphism $h:(\delta, Q) \rightarrow\left(\delta^{\prime}, Q^{\prime}\right)$ was a K-morphism $h: Q \rightarrow Q^{\prime}$ preserving the dynamics

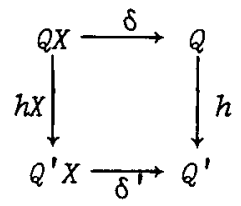

(we call such an $h$ a dynamorphism).

We then saw that if the forgetful functor $\operatorname{Dyn}(X) \rightarrow K$ has a left adjoint (we then call $X$ an input process), forming for each $K$-object $Q$ a free dynamics $Q \mu_{0}:\left(Q X^{巴}\right) X \rightarrow Q X^{巴}$ with 'insertion of the generators' $Q n: Q \rightarrow Q X^{Q}$, we could provide a reachability theory broad enough to encompass sequential machines, linear and group machines, and tree automata. The reachability map ${ }^{2}$ of an $X$-dynamics $(Q, \delta)$ equipped with initial state map $\tau: I \rightarrow Q$ is the unique dynamorphic extension $r: I X^{\Theta} \rightarrow Q$ of $\tau$ :
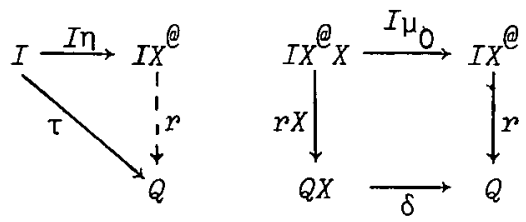

On the other hand, if $\operatorname{Dyn}(X) \rightarrow K$ has a right adjoint (we call $X$ an output process) forming for each K-object $Y$ a cofree dynamics $Y L:\left(Y X_{\varrho}\right) X \rightarrow Y X_{\varrho}$ with 'evaluation' $Y \Lambda: Y X_{\varrho} \rightarrow Y$, we could provide an

1 A semiring is unitary and satisfies all the ring axioms save that it need not. have additive inverses.

2 For convenience, we use 'map' and 'morphism' as synonyms in this paper. 
observability theory broad enough to emcompass sequential machines, and linear and group machines. The observability map of an $X$-dynamics $(Q, \delta)$ equipped with output map $B: Q \rightarrow Y$ is the unique dynamorphic coextension $\sigma: Q \rightarrow Y X_{\varrho}$ of $B:$

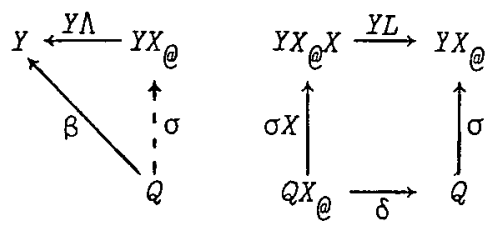

In particular, we know that if $X$ is an adjoint process - that is, $X$ has a right adjoint while $K$ has countable products and coproducts - then it is both.an input and output process. Since this includes the case where $K$ is a closed category with countable products and coproducts and $X=-\otimes X_{0}$ for some fixed object $x_{0}$ of $k$, we recapture the theory of Goguen [10] and Ehrig et al. [8].

In this paper, we undertake the analogous task for the right hand sides of (1), (2), and (3), asking for conditions on a functor $T$ which will enable $Q T$ to serve as an "object of fuzzy states", so that we may give a general theory of nondeterministic automata as systems with dynamics

$$
Q X \rightarrow Q T
$$

where $X$ is an input or output process, and $T$ is an appropriate functor. In [8, Chapter 6], the theory of pseudoclosed categories is advanced as the setting for nondeterministic machines with process $-\otimes X_{0}$; and we shalI see in Section 3 that our general theory does indeed include the Ehrig approach.

It would be aesthetically appealing to subsume dynamics $Q X \rightarrow Q T$ in the form $Q X \rightarrow Q$ of the theory of $[1,4]$. To do this, we need a new category $K_{T}$ with the same objects as $K$, but with a $K_{T}$-morphism $f: A \rightarrow B$ (note the single-headed arrow) being in reality a K-morphism $f: A \rightarrow B T$, so that a fuzzy dynamics is given by a $K_{T}$-morphism $Q X \rightarrow Q$. To be a category, $K_{T}$ must be equipped with identities and composition. Now, in each of our examples (1), (2), and (3), we have a map which allows to regard a 'pure' state as a 'fuzzy' state: 


$$
\begin{aligned}
& Q \rightarrow 2^{Q}: q \mapsto\{q\}, \\
& Q \rightarrow Q P: q \mapsto \varepsilon_{q} \text { where } \varepsilon_{q}\left(q^{\prime}\right)=1 \text { if } q^{\prime}=q \text { and is otherwise } 0, \\
& Q \rightarrow R^{(Q)}: q \mapsto \varepsilon_{q} \text { where } \varepsilon_{q}\left(q^{\prime}\right)=1 \text { if } q^{\prime}=q \text { and is otherwise } 0 .
\end{aligned}
$$

Each of these is of the form $Q e: Q-Q$, and we require, then, that the identity morphisms $Q e$ of $K_{T}$ generalize this role of letting us interpret pure states as particular examples of fuzzy states. Then for a collection of maps

$$
K(A, B T) \times K(B, C T) \rightarrow K(A, C T):(A \stackrel{\alpha}{\longrightarrow} B, B \stackrel{\beta}{\longrightarrow} C) \mapsto A \stackrel{\beta 0 \alpha}{\longrightarrow} C
$$

to be composition for $K_{T}$ it must satisfy the usual rules

$$
\begin{aligned}
& (\gamma \circ \beta) \circ \alpha=\gamma \circ(\beta \circ \alpha), \\
& \alpha \circ A e=\alpha=B e \circ \alpha .
\end{aligned}
$$

However, we require one more condition to make $e$ consistent with our interpretation - namely

$$
\beta \circ f^{\Delta}=\beta \cdot f \text { for } f: A \rightarrow B, \beta: B \rightarrow C,
$$

where we adopt the notation

$$
A \stackrel{\Delta}{\stackrel{\Delta}{\longrightarrow}} B=A \stackrel{f}{\longrightarrow} B \stackrel{B e}{\longrightarrow} B T
$$

for $f: A \rightarrow B$ "viewed as a relation" $A \rightarrow B$.

With this, we may give a formal definition and check it for $Q T=2^{Q}$, leaving the other cases to the reader.

DEFINITION 7. Let $T$ be a mapping $O b j(K) \rightarrow O b j(K)$, and write $\alpha: A-B$ for $\alpha: A \rightarrow B T$. Let, then, o be an associative "composition of fuzzy relations"; and let $A e: A \rightarrow A T$ for each $A$ in $K$ satisfy

$$
B \circ f^{\triangle}=\beta \cdot f
$$

as well as

$$
C e \circ \beta=\beta
$$

for each $f: A \rightarrow B$ (where $f^{\Delta}=B e \cdot f$ ) and each $\beta: B-C$. Then we 
call $T=(T, \circ, e)$ a fuzzy theory over $K$. (It is a consequence (Observation 3 , Section 2 ) of these axioms that $T$ can be made into a functor $K \rightarrow K$. )

The Kleisli category $K_{T}$ of $T$ has the same objects as $K$, "relations" $A-B$ as morphisms, - for composition, and the $A e: A-A$ as identities. That $K_{T}$ is a category follows from the axioms since

$$
\alpha \circ A e=\alpha \circ\left(i \alpha_{A}\right)^{\Delta}=\alpha \cdot i d_{A}=\alpha .
$$

(Note: We will use "id" exclusively for $K$ identities since $K_{T}$ identities are easily denoted with $e$.)

The concept of "monad" or "triple" is well-established in category theory ([13, Chapter V; 2, Chapter 10]) and is entirely coextensive with our notion of fuzzy theory (as is proved in [14, 1.3.18]). If $S$ is the monad corresponding to the fuzzy theory $T, K_{T}$ is the well-known "Kleisli category of $S$ " first defined by Kleisli [12]. While a knowledge of monad theory is certainly relevant, it is in no way essential to comprehension of this paper.

We further develop the concept of a fuzzy theory in Section 2. In the remainder of the present section, we develop the theory of nondeterministic sequential machines in such a way as to motivate the general theory of the subsequent sections.

EXAMPLE 8. The theory of nondeterministic sequential machines corresponds to $T=2^{(-)}$with

$$
\text { Qe }: q \mapsto\{q\} \text {, }
$$

while for $\alpha: A-B, \beta: B-C$, we have $B \circ \alpha: A \rightarrow 2^{C}$ defined by

$$
\beta \circ \alpha(a)=U\{\beta(b) \mid b \in \alpha(\alpha)\} \text {. }
$$

The reader may check the category axioms. Here we verify $\beta \circ f^{\wedge}=\beta \cdot f$ :

$$
\begin{aligned}
B \circ(B e \cdot f)(a) & =\{\beta(b) \mid b \in B e \cdot f(a)\} \\
& =\beta(f(a)) \text { since } B e \cdot f(a)=\{f(a)\} \\
& =\beta \cdot f(a) .
\end{aligned}
$$

10. Consider a nondeterministic sequential machine with initial 
"state" $\tau$ in $2^{Q}$, with dynamics $\delta: Q \times X_{0} \rightarrow 2^{Q}$, and with output map $B: Q \rightarrow\{0,1\}$. It is usual [15] to simulate such a nondeterministic sequential machine by a deterministic sequential machine with state-space $2^{Q}$, and with

initial state map $\tau: I+2^{Q}$, for some one-element set $I$, dynamics

$$
\bar{\delta}: 2^{Q} \times X_{0}+2^{Q}, \quad(p, x) \mapsto \underset{q \in p}{U} \delta(q, x),
$$

and

output map $\beta^{\#}: 2^{Q} \rightarrow\{0,1\}$, where $\beta^{\#}(p)=1 \Leftrightarrow \beta(q)=1$ for some $q \in p$.

We can express this in a more algebraic way:

$$
\begin{aligned}
\bar{\delta}(p, x) & =U\{\delta(q, x) \mid q \in p\} \\
& =U\{\delta(r) \mid r=(q, x) \text { for some } q \in p\} \\
& =\delta \circ Q \lambda(p, x),
\end{aligned}
$$

where we recall the form of $\circ$ from (9), and define $Q \lambda: 2^{Q} \times X_{0} \rightarrow Q \times X_{0}$ by

$$
Q \lambda: 2^{Q} \times X_{0} \rightarrow 2^{Q \times X} 0,(p, x) \mapsto\{(q, x) \mid q \in p\}
$$

11. Thus the passage from the fuzzy $\delta$ to the corresponding deterministic $\bar{\delta}$ may be expressed both in terms of the fuzzy theory composition and the map $Q \lambda$. In Section 3, we shall establish the properties $\lambda$ must satisfy to play this role for a given process $X$ and fuzzy theory $T$ - suitable $\lambda$ 's are called distributive zaws.

To analyze $\beta^{\#}$ more carefully, we first recall

DEFINITION 12. A complete semilattice is a partially ordered set in which every subset has a supremum. A finite semilattice is a complete semilattice whose underlying set is finite. A semilattice homomorphism is a supremum-preserving map.

We now observe that the output set $\{0,1\}$ of our machine is a complete semilattice under the supremum operation $\max$, while the state set $2^{Q}$ of our deterministic simulator is a complete semilattice under the 
supremum operation of union. We now note the commutativity of

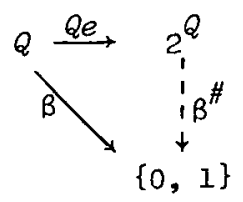

In fact, it is easily seen that:

13. $\beta^{\#}$ is the unique homomorphic extension $\left(2^{Q}, u\right) \rightarrow(\{0,1\}, \max )$ of $\beta$. In Section 4 , we introduce T-deciders $(Q, \xi)$ as the generalization for a fuzzy theory $T$ of the complete semilattices associated with $T=2^{(-)}$, whereas for $T$ as in $(2),(Q, \xi)$ is a 'generalized' convex set. $Q T$ is itself always a decider via the canonical map $Q n: Q T T \rightarrow Q T$ defined by

$$
Q m=\left(Q T \stackrel{i \mathrm{~d}_{Q T}}{\longrightarrow} Q T\right) \circ\left(Q T T \stackrel{i \mathrm{~d}_{Q T T}}{\longrightarrow} Q T T\right) .
$$

For example, for $T$ as in $(1),\left(2^{Q}, u\right)=\left(2^{Q}, Q m\right)$ since $Q m: 2^{\left(2^{Q}\right)} \rightarrow 2^{Q}$ assigns to each family its union, as is routinely checked.

This special case of free $T$-deciders - $(Q T, Q m)$ - (see Theorem 7 , Section 4 ) has been implicit in the literature of nondeterministic and stochastic automata theory. It is the contribution of this paper to show, in Section 7, how more general $T$-deciders are used to formulate the fuzzy minimal realization problem.

Returning to our nondeterministic dynamics $\bar{\delta}$, we note on taking $\bar{Q}=2^{Q}$ that it provides an example of:

DEFINITION 15. A complete semilattice with operators indexed by $x_{0}$ (we write FSO for a finite such structure) is a complete semilattice $(\bar{Q}, \xi)$ together with a function $\bar{\delta}: \bar{Q} \times X_{0} \rightarrow \bar{Q}$ such that for each $x$ in $X_{0}, \bar{\delta}(-, x): \bar{Q} \rightarrow \bar{Q}$ preserves suprema.

16. It is this notion of an object which supports both a T-decider structure (semilattice, in this case) and a dynamic structure in a compatible way which motivates the theory of $\lambda$-algebras to be developed in Section 5 . 
17. The nondeterministic machine

$$
\left(\tau: I \rightarrow 2^{Q}, \delta: Q \times X_{0} \rightarrow 2^{Q}, B: Q \rightarrow\{0,1\}\right)
$$

will yield the notion of a $\lambda$-machine in Section 5; while the expanded machine $\left(\tau: I \rightarrow 2^{Q}, \bar{\delta}: \bar{Q} \times X_{0} \rightarrow \bar{Q}, \bar{B}: \bar{Q} \rightarrow\{0,1\}\right)$ - in which $\bar{\delta}$ is a complete semilattice with operators indexed by $X_{0}$ (that is, a $\lambda$-algebra) while $\bar{B}$ is a homomorphism - will yield the notion of an implicit $\lambda$-machine. It is a major observation of this paper that fuzzy machine theory supports two distinct kinds of automaton (which coincide in the deterministic case).

18. In Section 7, we shall give the general theory of minimal realization of suitably defined response functions $f$. We shall find that each $f$ has a minimal implicit $\lambda$-machine which realizes it uniquely up to isomorphism, but that nonisomorphic $\lambda$-machines may be minimal and yet realize the same $f$. To illustrate this distinction, we shall give examples from, and an outline of, the theory of minimal realization for nondeterministic sequential machines.

\section{Fuzzy theories}

Much has been written about fuzzy set theory ([18], [9]; see also [11] and the extensive bibliography there). From this point of view, the fuzzy theories of Definition 7, Section 1, provide very general categories of fuzzy relations. Our heuristics for the fuzzy category concepts are as follows: $A T$ is the "cloud of fuzzy states over $A$ ". A morphism $\alpha: A \rightarrow B=\alpha: A \rightarrow B T$ is "a fuzzy relation from $A$ to $B$ ". $A e$ is the "pure state" map.

Now fix a fuzzy theory $T=(T, e, 0)$ over $K$. We establish a few general properties, and then provide several examples of fuzz theories. (For further properties and examples, see [14, 4.3].)

CONTRACTION PRINCIPLE 1. id ${ }_{A T}: A T \rightarrow A T$ may be regarded as the "relation" id $A T: A T \rightarrow A$.

OBSERVATION 2. Each morphism $\alpha: A \rightarrow B T$ admits a canonical extension $\alpha^{\#}$, 


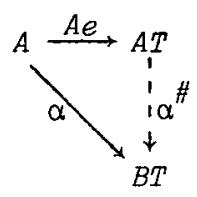

defined by $\alpha^{\#}=\alpha \circ$ id $_{A T}$ (cf. 13, Section 1), such that $\alpha^{\#} \cdot A e=\alpha$.

Proof. By the fuzzy theory axioms we have

$$
\alpha^{\#} \cdot A e=\left(\operatorname{\alpha id}_{A T}\right) \circ(A e)^{\Delta}=\alpha \circ\left(\mathrm{id}_{A T^{\circ}} \circ(A e)^{\Delta}\right)=\alpha \circ A e=\alpha .
$$

OBSERVATION 3. $T: K \rightarrow K$ becomes a functor when, for $f: A \rightarrow B$, we define $f T: A T \rightarrow B T$ by $f T=\left(f^{\Delta}\right)^{\#}$; that is, $f T=f^{\Delta} \circ i \mathrm{~d}_{A T}=(B e \cdot f) \circ \mathrm{id}_{A T}$.

Proof. (i) id ${ }_{A} T=A e \circ{ }^{i d} A T=i d_{A T}$.

(ii) Given $g: B \rightarrow C$,

$$
\begin{aligned}
(g \cdot f) T= & (g \cdot f)^{\Delta} \circ{i d_{A T}}_{A}\left(g^{\Delta} \cdot f\right) \circ i_{A T}=g^{\Delta} \circ f^{\Delta} \circ i d_{A T}=g^{\Delta} \circ f T= \\
& =g^{\Delta} \circ\left(i d_{B T} \cdot f T\right)=g^{\Delta} \circ i d_{B T} \circ(f T)^{\Delta}=g T \circ(f T)^{\Delta}=g T \cdot f T .
\end{aligned}
$$

OBSERVATION 4. Given $\alpha: A \rightarrow B, \beta: B \rightarrow C, \beta \circ \alpha=\beta^{\#} \cdot \alpha$. In particular, for $\alpha: A \rightarrow B$ and $f: B \rightarrow C, f^{\Delta} \circ \alpha=f T \cdot \alpha$.

Proof. $\beta \circ \alpha=\beta \circ i d_{B T} \circ \alpha^{\Delta}=\beta^{\#} \circ \alpha^{\Delta}=\beta^{\#} \cdot \alpha$.

With these sample properties, we now turn to a number of examples, in each of which we let $K$ be the category set of sets and functions. We first summarize our observations in Section 1 on the 'classic' case:

EXAMPLE 5. Let $A T=2^{A}$ so that $\alpha: A-B$ is the familiar concept of rezation via $a \alpha b \Leftrightarrow b \in \alpha(a)$. The singleton map $A e, a \mapsto\{a\}$, represents the equality relation. $\beta \circ \alpha$ is the usual composition, $\left(\beta^{\circ} \alpha\right)(a)=\{c \in C \mid$ there exists $b$ in $B$ with $b \in \alpha(a)$ and $c \in \beta(b)\}$. $K_{T}$ is then the usual category of sets and relations. 
id $_{A T}$, as in Contraction Principle 1 , is the elementhood relation:

$$
S \operatorname{id}_{A T} a \Leftrightarrow a \in S \text {. }
$$

$\alpha^{\#}(S)=U\{\alpha(s) \mid s \in S\}$.

$f T: A T \rightarrow B T$ is the direct image map, $S \mapsto\{f(a) \mid a \in S\}$.

$A m: A T T \rightarrow A T$ (as in 13, Section 1) is the union map.

$S \mapsto U S=\{a \in A \mid a \in S$ for some $S \in S\}$.

The same constructions work if AT is restricted to "finite subsets", "non-empty subsets", or "finite, non-empty subsets".

EXAMPLE 6. Let $A T=A P$ as in (2), Section 1. $\alpha: A \rightarrow B$ corresponds to an $A-b y-B$ column-stochastic matrix (we think of $A$ as indexing columns and $B$ as indexing rows). $(A e)(a)$ is the stochastic column with entry $I$ in its ath place. $B \circ \alpha$ is the usual matrix product.

EXAMPLE 7. Let $A T=R^{(A)}$ as in (3), Section 1. Then $\alpha: A \rightarrow B$ is a column-finite matrix of entries from $R$ and a fuzzy theory is constructed in the same way as in Example 6. The "finite subsets" version of Example 5 is recovered by taking $R$ to be a two-element semiring (not a ring!) with the usual mod 2 addition but with conjunction as multiplication.

EXAMPLE 8 ([9, Section 6]). Let $L$ be a complete lattice which is completely distributive in the sense that for each $x$ in $L, x \vee(-)$ preserves infima and $x \wedge(-)$ preserves suprema. Set $A T=L^{A} . A e$ is the "crisp singleton map"; that is, $(A e)(a)$ maps $a^{\prime}$ to the greatest element of $L$ when $a^{\prime}=a$ and to the least element otherwise. $\alpha: A \rightarrow B$ is Goguen's L-fuzzy relation. $\beta \circ \alpha$ is defined by

$$
[(\beta \circ \alpha)(\alpha)](c)=v\{[\beta(b)](c) \wedge[\alpha(a)](b) \mid b \in B\}
$$

and the proof that $\circ$ is associative requires complete distributivity. Example 5 (with all subsets) is recovered by taking $L$ to be the twoelement lattice. With $L$ the unit interval, $K_{T}$ is Zadeh's category of fuzzy relations [18].

EXAMPLE 9. Let $M$ be an arbitrary monoid "of credibility values", 
and take $A T=A \times M$. If $\alpha: A-B, " \alpha(\alpha)=b$ with credibility $x "$ if $\alpha(a)=(b, x)$. $(A e)(a)=(a, 1)$. If $\alpha(a)=(b, x)$ and $B(b)=(c, y)$ then $(\beta \circ \alpha)(a)=(c, x y)$.

EXAMPLE 10. Let $A T=A^{*}$, the free monoid of all strings in $A$. If $\alpha: A \rightarrow B, \alpha(a)=b_{1} \ldots b_{n}$ expresses a "voting preference for choice $a$ ". The empty word represents "abstention". $(A e)(a)=a$, the string of length 1 . If $\alpha(a)=b_{1} \ldots b_{n},(\beta \circ \alpha)(a)=\beta\left(b_{1}\right) \ldots \beta\left(b_{n}\right)$.

\section{Distributive laws}

We motivated the construction of the Kleisli category $K_{T}$ of Definition 7 , Section 1 , by suggesting that nondeterministic dynamics $Q X \rightarrow Q T$ in the category $K$ be viewed as normal dynamics

$$
Q X \rightarrow Q
$$

in $K_{T}$. Now while the functor $X: K \rightarrow K$ gives us an object function $Q \mapsto Q X$ in $K_{T}$, it does not give us a map on morphisms in $K_{T}$, since $(A \rightarrow B T) X$ is not of the form $A X \rightarrow B X=A X \rightarrow B X T$. Our task, then, is to find a "lifting" $\bar{X}$ of $X$ :

$$
A \stackrel{g}{\longrightarrow} B \mapsto A X \stackrel{g \bar{X}}{\longrightarrow} B X
$$

OBSERVATION 1. $K$ may be viewed as a subcategory of $K_{T}$ via "a morphism is a relation"; that is, $f \mapsto f^{\Delta}$.

Proof. We have $\left(\mathrm{id}_{A}\right)^{\Delta}=A e$ and, given $f: A \rightarrow B$ and $g: B \rightarrow C$, we further have $(g \cdot f)^{\Delta}=g^{\Delta} \cdot f=g^{\Delta} \circ f^{\Delta}$.

DEFINITION 2. By a lifting of a functor $X: K \rightarrow K$ to $k_{T}$ we mean a functor $\bar{X}: K_{T} \rightarrow K_{T}$ such that

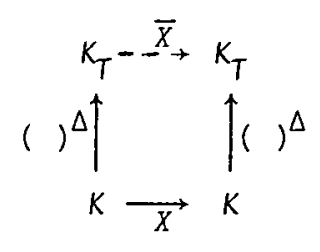

is a commutative square of functors. 
Note now that if $\bar{X}$ is such a lifting, we have

$$
\begin{aligned}
\alpha \bar{X} & =\left(i d_{B T} \cdot \alpha\right) \bar{X} \\
& =\left(i d_{B T}{ }^{\circ \alpha}\right) \bar{X} \\
& =i d_{B T^{\Delta}} \bar{X} \circ \alpha^{\Delta \bar{X}} \text { since } \bar{X} \text { is a functor } \\
& =i d_{B T} \bar{X} \circ(\alpha X)^{\Delta} \text { by Definition 2 } \\
& =i d_{B T} \bar{X} \cdot \alpha X .
\end{aligned}
$$

Summarizing, then:

LEMMA 3. If $X$ is as in Definition 2, then for all $\alpha: A \rightarrow B$, $\alpha \bar{X}: A X \rightarrow B X$ is given by

$$
\alpha \bar{X}=A X \stackrel{\alpha X}{\rightarrow} B T X \stackrel{B \lambda}{\longrightarrow} B X T,
$$

where

$$
B \lambda: B T X-B X=i d_{B T^{X}}
$$

NOTE. $B(T X)$ means $(B T) X$, not $(B X) T$, in accordance with our notation for the action of functors.

The lema raises the question: "Under what conditions on $\lambda$ does $\alpha \bar{X}=B \lambda \cdot \alpha X$ define a lifting of $X ?^{\prime \prime}$ The appropriate definition and proposition are:

DEFINITION 4. A distributive law of $X$ over $T$ is an assignment to each object $A$ of $K$ of a morphism $A \lambda: A T X \rightarrow A X T$ such that the following two diagrams commute for all $A$ and $\alpha: A \rightarrow B$.
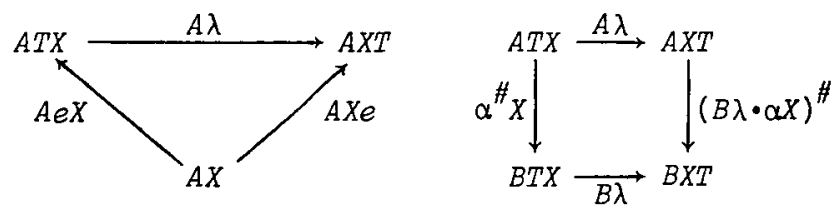

This definition can be shown to be coextensive with the distributive laws between monads of [6] - where the terminology is motivated by the distributive law of ring theory.

PROPOSITION 5. The correspondence, Lemma 3, establishes a bijection between liftings $\bar{X}$ as in Definition 2 and distributive laws as in 
Definition 4 .

Proof. If $\bar{X}$ is a lifting and $\lambda$ is defined by $B \lambda=i_{B T} \bar{X}$, then

$$
\begin{aligned}
A \lambda \cdot A e X=\operatorname{id}_{A T} \bar{X} \bullet A e X=\operatorname{id}_{A T} \bar{X} \circ(A e X)^{\Delta}=i_{A T} \bar{X} \circ(A e)^{\Delta} \bar{X} & \\
& =\left(i_{A T} \circ(A e)^{\Delta}\right) \bar{X}=(A e) \bar{X}=A X e
\end{aligned}
$$

since $\bar{X}$ preserves identities. Moreover,

$$
\begin{aligned}
(B \lambda \cdot \alpha X)^{\#} \cdot A \lambda & =\left(\alpha \bar{X} \circ i d_{A X T}\right) \cdot\left(i_{A T} \bar{X}\right) \quad \text { by the argument preceding Lemma } 3 \text { and } \\
& =\alpha \bar{X} \circ{i d_{A X T}} \circ\left(\operatorname{id}_{A T} \bar{X}\right)^{\Delta}=\alpha \bar{X} \circ\left(\text { id }_{A T} \bar{X}\right)=\left(\alpha_{A i d_{A T}}\right) \bar{X} \\
& =\alpha^{\#} \bar{X}=B \lambda \cdot \alpha^{\#} X .
\end{aligned}
$$

Now let $\lambda$ be a distributive law and define $\bar{X}$ by $\alpha \bar{X}=B \lambda \cdot \alpha X$. That $A e \vec{X}=A \lambda \cdot A e X=A X e$ is immediate. The second diagram in Definition 4 reduces to

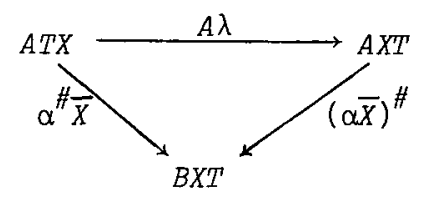

Since $\beta \circ \alpha=\beta^{\#} \cdot \alpha$ by Observation 2 , Section 2 , we have

$$
\begin{aligned}
(\beta \circ \alpha) \bar{X} & =C \lambda \cdot\left(\beta^{\#} \cdot \alpha\right) X=C \lambda \cdot \beta^{\#} X \cdot \alpha X=\beta^{\#} \bar{X} \cdot \alpha X=(\beta \bar{X})^{\#} \cdot A \lambda \cdot \alpha X \\
& =(\beta \bar{X})^{\#} \cdot(\alpha \bar{X})=(\beta \bar{X}) \circ(\alpha \bar{X}) .
\end{aligned}
$$

Thus $\bar{X}$ is indeed a functor. For $\bar{X}$ thus defined from $\lambda$ we have

$$
\left(\mathrm{id}_{A T}\right) \bar{X}=A \lambda \cdot\left(\mathrm{id}_{A T}\right) X=A \lambda \text {, }
$$

which does indeed recover $\lambda$.

To see that $\bar{X}$ is a lifting of $X$, we note that

$$
\alpha^{\Delta} \bar{X}=B T \lambda \cdot \alpha^{\Delta} X=B T \lambda \cdot B T e X \cdot \alpha X=B T X e \cdot \alpha X=(\alpha X)^{\Delta} \text {. }
$$

In the future we shall write $X_{\lambda}$ (rather than $\bar{X}$ ) for the lifting corresponding to $\lambda$. 
The following proposition is sensibly stated now although its proof relies on results to be independently established below.

PROPOSITION 6. Let $T$ be a fuzzy theory over set and let $x=-\times x_{0}:$ Set $\rightarrow$ Set . Then

$$
\begin{aligned}
& Q T \times X_{0} \stackrel{Q \lambda}{\longrightarrow}\left(Q \times X_{0}\right) T \\
& (p, x) \mapsto\left(\operatorname{in}_{x} T\right)(p)
\end{aligned}
$$

(where in in $\left._{x} Q \rightarrow Q \times X_{0}, q \mapsto(q, x)\right)$ is a distributive low of $X$ over $T$.

Proof. The proof that $A X e=A \lambda \cdot A e X$ is clear since $e:$ id $\rightarrow T$ is a natural transformation (see (2), Section 6 below). To prove the second distributive law axiom it suffices to prove that (II) commutes for every $x$ in $x_{0}$;

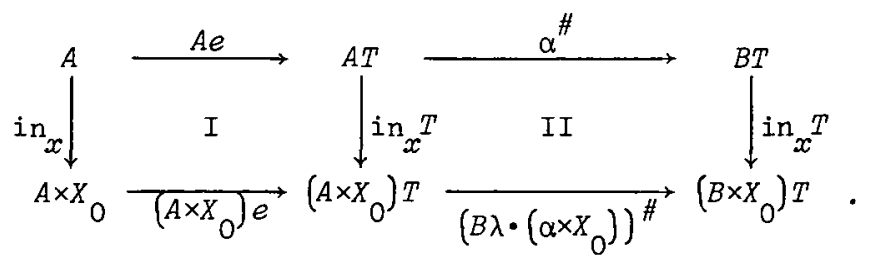

I always commutes ((2), Section 6). Using Observation 2, Section 2, and the definition of $\lambda$, the outer rectangle commutes; that is, (II) commutes preceded by $A e$. It follows from Theorem 7, Section 4, that (II) commutes.

The value of distributive laws for machine theory is given by the following:

PROPOSITION 7. Let $\lambda$ be a distributive law and let $\left(A X^{Q}, A \mu_{0} ; A n\right)$ be a free X-dynamics over $A$. Then $\left(A X^{Q},\left(A \mu_{0}\right)^{\Delta} ;(A n)^{\Delta}\right)$ is a free $X_{\lambda}$-dynamics over $A$. Thus if $X$ is an input process, so is each $X_{\lambda}$.

Proof. Let $\left(Q, \delta: Q X_{\lambda} \rightarrow Q\right)$ be an $X_{\lambda}$-dynamics and let $\alpha: A \neg Q$. Not surprisingly, we use the associated $X$-dynamics as in 10 , Section 1 recall the formula $\delta=\delta \circ Q \lambda=\delta^{\#} \cdot Q \lambda: Q T X \rightarrow Q T$. Specifically, for 
$\psi: A X^{@}-Q$ compare

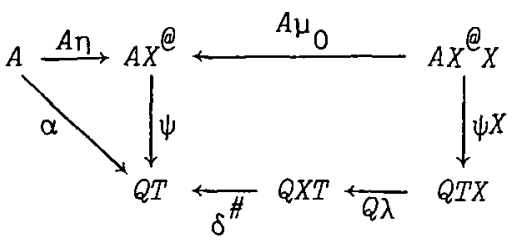

with

(9)

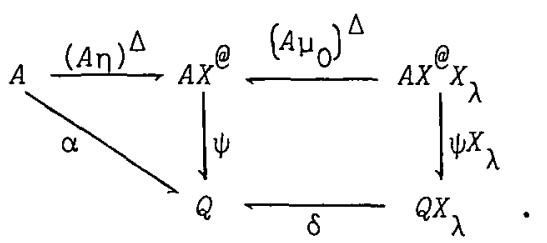

Since

$$
\begin{aligned}
\psi \circ\left(A_{\eta}\right)^{\Delta} & =\psi \cdot A \eta, \\
\psi \circ\left(A \mu_{0}\right)^{\Delta} & =\psi \cdot A \mu_{0},
\end{aligned}
$$

and

$$
\delta \circ \psi X_{\lambda}=\delta \circ(Q \lambda \cdot \psi X)=\delta^{\#} \cdot Q \lambda \cdot \psi X,
$$

we have "( 8 ) commutes, for unique $\psi$, in $K$ " if and only if "(9) commutes, for unique $\psi$, in $k_{T}$ ".

EXAMPLE 10. As a special case of Proposition $6, \lambda$ as in 10 , Section 1, is a distributive law.

EXAMPLE 11. Let $\Omega$ be the operator domain with one binary operation and let $A X=A \times A$ be the corresponding tree automaton input process in Set (see [1]). Let $T$ be as in Example 5, section 2. Then

$$
\begin{aligned}
& 2^{A} \times 2^{A} \stackrel{A \lambda}{\longrightarrow} 2^{A \times A} \\
& \left(S_{1}, S_{2}\right) \mapsto S_{1} \times S_{2}
\end{aligned}
$$

is a distributive law. This example generalizes easily to arbitrary operator domains.

We can now sketch the approach to fuzzy machine theory of [8]. (The 
reader unfamiliar with closed and monoidal categories may consult [2].)

Ehrig et al. work only in a closed category $K$ with countable products and coproducts, and use only the adjoint process $-\otimes X_{0} \cdot$ Then, rather than construct a Kleisli category $K_{T}$ as we did in (2), Section 1, they consider any category $L$ which is pseudoclosed with respect to $K$; that is:

(1) L has the same objects as the closed category $K$ and is structured with a pair of functors

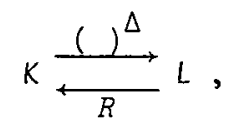

where $R$ is right adjoint to ()$^{\Delta}$;

(2) $L$ is a monoidal category and ()$^{\Delta}$ is a monoidal functor; that is

$$
(f \otimes g)^{\Delta}=f^{\Delta} \otimes^{\prime} g^{\Delta} .
$$

The condition ( 1 ) is equivalent to our conditions defining a fuzzy theory $T$. Given (1), define $K T=K \Lambda_{R}$ and let $e$ and $\circ$ be induced by the identity and composition of $L ; L$ may then be identified with $K_{T}$. Conversely, given $T$, set $B^{\triangle} R=B T ;$ the desired adjointness is

$$
\frac{A^{\Delta}-B}{A \rightarrow B T}
$$

The point of (2) is to ensure that $-\otimes X_{0}$ lifts to a functor on $L$. This, then, corresponds to our distributive laws, in view of our Proposition 5. In short:

OBSERVATION 12. The Ehrig theory [8, Chapter 6] is a special case of ours, applying when $X: K \rightarrow K$ has the special form $-\otimes X_{0}$ in a closed category.

\section{Deciders}

For the balance of this paper, $T$ denotes a fuzzy theory in an 
arbitrary category $K$.

Our task now is to define a $T$-decider as the generalization of the complete semilattices which, in 13 , Section 1 , we associated with the 'classical' fuzzy theory $T$, with $T=2^{(-)}$, of Example 5, Section 2. Clearly, the supremum $\xi(\{q\})$ of a one-element subset of a complete semilattice $Q$ is simply $q$ - and we may rewrite this equality as $\xi \cdot Q e=i d_{Q}$. More subtly, recall that in Example 5, Section 2, we had $\alpha^{\#}(S)=U\{\alpha(s) \mid s \in S\}$. The crucial property of suprema that $" \xi\left(S_{a}\right)=\xi\left(\bar{S}_{a}\right)$ for each $a$ in $A$ implies that $\xi\left(U S_{a}\right)=\xi\left(U \bar{S}_{a}\right)$ " may then be re-expressed by stating that if $\alpha, \beta: A \rightarrow 2^{Q}$ satisfy $\xi \cdot \alpha=\xi \cdot \beta$, then we have $\xi \cdot \alpha^{\#}=\xi \cdot \beta^{\#}$.

This yields the general definition:

DEFINITION 1. A $T$-decider is a pair $(Q, \xi)$ with $\xi: Q T \rightarrow Q$ a $K$-morphism subject to the conditions

(i) $\xi \cdot Q e=i d_{Q}$; and

(ii) whenever $\alpha, \beta: A \rightarrow Q T$ are such that $\xi \bullet \alpha=\xi \bullet \beta$, then $\xi \cdot \alpha^{\#}=\xi \cdot \beta^{\#}$.

The heuristic meaning of the first axiom is clear. The second axiom asserts that the structure of $T$ imposes some 'deterministic' ways of building fuzzy states from other fuzzy states which must be respected by $\xi$; further intuition may be inferred from the following examples:

EXAMPLE 2. Let $T$ be as in Example 10, Section 2. If $M$ is a monoid, the map $\xi: M^{*} \rightarrow M$ which realizes formal multiplication is a decider and, conversely, every decider is a monoid with multiplication $(x, y) \mapsto \xi(x y)$; the two concepts are the same. Axiom (ii) may be formulated as "whenever $\left(w_{1}, \ldots, w_{n}\right),\left(\bar{w}_{1}, \ldots, \bar{w}_{n}\right)$ are $n$-tuples of elements of $Q^{*}$ such that $\xi\left(w_{i}\right)=\xi\left(\bar{w}_{i}\right)$, then $\xi\left(w_{1} \ldots w_{n}\right)=\xi\left(\bar{w}_{1} \ldots \bar{w}_{n}\right) \cdot "$

EXAMPLE 3. For $T$ as in Example 9, Section 2, (ii) asserts that "if $\xi(q, x)=\xi(\bar{q}, \bar{y})$ then $\xi(q, x z)=\xi(\bar{q}, \bar{y} z)$ for all $z$." A T-decider 
is an $M$-set: the more standard equivalent axioms being (i) and $" \xi(q, x y)=\xi(\xi(q, x), y) . "$

EXAMPLE 4. Let $T$ be as in Example 6, Section 2. Every convex subset of a real linear space is a decider, where $\xi$ converts elements of QT (thought of as formal convex combinations) into actual convex

combinations. The arbitrary stochastic decider is not of this form since

$$
\xi:\{x, y\} T \rightarrow\{x, y\}, \lambda x+\mu y \mapsto\left\{\begin{array}{l}
x \text { if } \lambda \neq 0, \\
y \text { if } \lambda=0,
\end{array}\right.
$$

satisfies the axioms. Axiom (ii) asserts that

$$
\xi\left(\lambda_{1} p_{1}+\ldots+\lambda_{n} p_{n}\right)=\xi\left(\lambda_{1} \bar{p}_{1}+\ldots+\lambda_{n} \bar{p}_{n}\right)
$$

for any $\left(\lambda_{1}, \ldots, \lambda_{n}\right)$ (all nonnegative and summing to 1 ), so long as $\xi\left(p_{i}\right)=\xi\left(\bar{p}_{i}\right)$.

In the language of monads, deciders are the well-known algebras over a monad. Deciders, then, are coextensive with universal algebra (see [2, Chapter 10], [13, Chapter 6], and [14]).

DEFINITION 5. If $(Q, \xi),(R, \theta)$ are $T$-deciders, a morphism $f: Q \rightarrow R$ is a T-homomorphism $f:(Q, \xi) \rightarrow(R, \theta)$ just in case we have

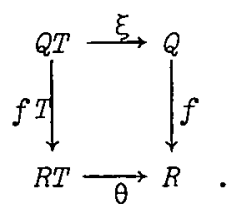

Since $T$ is a functor, $\operatorname{id}_{Q}:(Q, \xi) \rightarrow(Q, \xi)$ is a $T$-homomorphism and the composition of $T$-homomorphisms is again a $T$-homomorphism. Let $K^{\top}$ denote the category of $T$-deciders and $T$-homomorphisms.

The following fundamental result establishes a canonical free decider over any object. Recall ((14), Section 1) that

$$
A m=\left(A T \stackrel{i \mathrm{~d}_{A T}}{\longrightarrow} A\right) \circ\left(A T T \stackrel{\mathrm{id}_{A T T}}{\longrightarrow} A T\right)
$$

(which equals $\left(\text { id }_{A T}\right)^{\#}$ in the sense of observation, Section 2). 
THEOREM 7. (AT, Am) is a decider. Moreover, given any decider $(Q, \xi)$ and morphism $f: A \rightarrow Q$ there exists a zonique T-homomorphism $f^{\#}:(A T, A m) \rightarrow(Q, \xi)$,

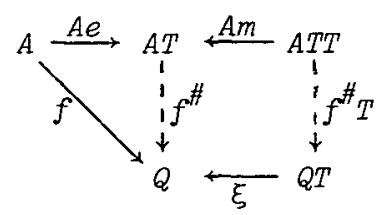

such that $f^{\#} \cdot A e=f$. The formuza for $f^{\#}$ is

$$
f^{\#}=A T \stackrel{f T}{\longrightarrow} Q T \stackrel{\xi}{\longrightarrow} Q .
$$

The notation is not ambiguous since, given $(B T, B m)$ and any $\alpha: A \rightarrow B T, \alpha^{\#}=\alpha \circ i_{A T}$, as in Observation 2, Section 2, is equal to $B m \cdot \alpha T$.

Proof. First, to check consistency of the \# notation, we have for $\alpha: A \rightarrow B T$,

$$
\alpha \circ i d_{A T}=i d_{B T} \circ \alpha^{\Delta} \circ i d_{A T}=i d_{B T} \circ \alpha T=\left(i d_{B T}\right)^{\#} \cdot \alpha T=B m \cdot \alpha T .
$$

We next show that $(Q T, Q m)$ is a decider. For axiom (i), we have

$$
\begin{aligned}
Q m \cdot Q T e & =\left(\mathrm{id}_{Q T}\right)^{\#} \cdot Q T e \\
& =i \mathrm{~d}_{Q T} \circ Q T e=i \mathrm{~d}_{Q T} .
\end{aligned}
$$

For (ii), if $Q m \cdot \alpha=Q m \cdot \beta$,

$$
\begin{aligned}
Q m \cdot \alpha^{\#} & =\left(i d_{Q T}\right)^{\#} \cdot \alpha^{\#}=i d_{Q T} \circ \alpha^{\#}=i d_{Q T} \circ \alpha \circ i d_{A T}=(Q m \cdot \alpha) \circ i d_{Q T} \\
& =(Q m \cdot \beta) \circ i d_{A T}=Q m \cdot \beta^{\#} .
\end{aligned}
$$

Next, we must show that $f^{\#}$ as in (8) is a T-homomorphism. Since $\xi \cdot$ id $_{Q T}=\xi=\operatorname{id}_{Q} \cdot \xi=\xi \cdot \xi^{\Delta}$ by Definition 1 (i), we may use Definition 1 (ii) to deduce that

$$
\xi \cdot\left(i_{Q T}\right)^{\#}=\xi \cdot\left(\xi^{\Delta}\right)^{\#}=\xi \cdot \xi T .
$$

Therefore, 


$$
\begin{aligned}
f^{\#} \cdot A m & =\xi \cdot f T \cdot A m=\xi \cdot\left(f^{\Delta} \circ A m\right) \quad(\text { recall Observation } 4, \text { Section 2) } \\
& =\xi \cdot\left(f^{\Delta} \circ i d_{A T}{ }^{\circ i d_{A T T}}\right)=\xi \cdot\left(f T \circ i d_{A T T}\right)=\xi \cdot\left(i d_{Q T}{ }^{\circ}(f T)^{\Delta_{\circ i d}}{ }_{A T T}\right) \\
& =\xi \cdot\left(i d_{Q T} \circ(f T)^{\Delta \#}\right)=\xi \cdot\left(i_{Q T}\right)^{\#} \cdot(f T)^{\Delta \#} \\
& =\xi \cdot \xi T \cdot f T T=\xi \cdot f^{\#} T .
\end{aligned}
$$

Moreover, $f^{\#} \cdot A e=\xi \cdot f T \cdot A e=\xi \cdot\left(f^{\Delta}\right)^{\#} \cdot A e=\xi \cdot f^{\Delta}=\xi \cdot Q e \cdot f=f$.

It only remains to verify uniqueness. Suppose $g:(A T, A m) \rightarrow(Q, \xi)$ is a T-homomorphism $-g \cdot A m=\xi \cdot g^{T}-$ such that $g \cdot A e=f$. Then

$$
\begin{aligned}
g & =g \cdot\left(\text { Aeoid }_{A T}\right)=g \cdot\left(i_{A T} \circ(A e)^{\Delta} \circ{ }_{A d}\right)=g \cdot\left(i_{A T} \circ A e T\right) \\
& =g \cdot\left(i_{A T}\right)^{\#} \cdot A e T=g \cdot A m \cdot A e T=\xi \cdot g T \cdot A e T \\
& =\xi \cdot(g \cdot A e) T=\xi \cdot f T=f^{\#} . \quad \square
\end{aligned}
$$

By Theorem $7, K_{T}$, the Kleisli category of "fuzzy relations" is isomorphic to the full subcategory of $K^{T}$ comprising all free deciders $(A T, A m)$, for

$$
(A e)^{\#}=i_{A T} \text { and }\left(\beta^{\circ} \alpha\right)^{\#}=\beta^{\#} \cdot \alpha^{\#}
$$

(since both $T$-homomorphisms are $\beta \circ \alpha$ when preceded by $A e$ ). As will be clarified in the remainder of the paper, many automata-theoretic constructions operating on objects in $K_{T}$ cannot be defined in $K_{T}$ but can be defined in the larger - and much better behaved - universe $K^{\top}$.

\section{Fuzzy machines}

For the rest of the paper, fix a functor $X: K \rightarrow K$ and a distributive law $\lambda: T X \rightarrow X T$ of $X$ over $T$. We now fulfill the promise of 16, Section 1 .

DEFINITION 1. A $\lambda$-aZgebra is a triple $(Q, \delta, \xi)$ with $(Q, \delta)$ an $X$-dynamics and $(Q, \xi)$ a $T$-decider in such a way that $\xi$ is an $X$-dynamorphism $(Q T, \delta T \cdot Q \lambda) \rightarrow(Q, \delta)$; that is, in such a way that we have 


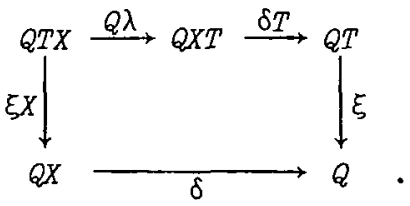

By a $\lambda$-homomorphism $f:(Q, \delta, \xi) \rightarrow\left(Q^{\prime}, \delta^{\prime}, \xi^{\prime}\right)$ between $\lambda$-algebras we mean a simultaneous dynamorphism and $T$-homomorphism.

EXAMPLE 2. Set $A T=A, A e=i \mathrm{~d}_{A}$, and $\beta \circ \alpha=\beta \cdot \alpha$. Then $T$ is a fuzzy theory in $K$, the identity theory. Clearly $K_{T}=K$. Moreover, if $(Q, \xi)$ is a $T$-decider for this theory, then axiom (i) of Definition 1, Section 4, stipulates that $\xi=\mathrm{id}_{Q}$. Thus we also have $K=K^{\top}$. Finally, $A \lambda=i_{A X}: A T X \rightarrow A X T$ is a distributive law of $X$ over $T$, and the category of $\lambda$-algebras is just $\operatorname{Dyn}(X)$.

If $K$ has an initial object 0 (for example, the empty set in set) then for arbitrary $T$ and $X$ defined by $A X=0, f X=i d_{0}$, the $\lambda$ defined by $A \lambda=i d_{0}$ is a distributive law of $X$ over $T$ and the category of $\lambda$-algebras may be identified with $K^{\top}$.

EXAMPLE 3. When $X=-x x_{0}:$ Set $\rightarrow$ Set and $\lambda$ is as in 10, Section $1,(\bar{Q}, \bar{\delta}, \xi)$ satisfies Definition $l$ if and only if $\bar{\delta}_{x}=\bar{\delta}(-, x):(\bar{Q}, \xi) \rightarrow(\bar{Q}, \xi)$ is a T-homomorphism for every $x$ in $X_{0}$. Thus the semilattices with operators discussed in Definition 15, Section 1 , are $\lambda$-algebras.

For the distributive law $Q \lambda:(p, x) \rightarrow$ in $_{x} T \cdot p$ of Proposition 6, Section 3, the diagram of Definition 1 says that

$$
\begin{aligned}
\bar{\delta}_{x} \xi(p) & =\xi \cdot \bar{\delta} T \cdot \text { in }_{x} T(p) \\
& =\xi \cdot\left(\bar{\delta} \cdot \text { in }_{x}\right) T(p) \\
& =\xi \cdot \bar{\delta}_{x} T(p) ;
\end{aligned}
$$

that is, each $\bar{\delta}_{x}$ is a T-homomorphism $(\bar{Q}, \xi) \rightarrow(\bar{Q}, \xi)$.

LEMMA 4. Let $K=$ Set. Then the image of a $\lambda$-homomorphism is a $\lambda$-subalgebra. More precisely, let $f:(Q, \delta, \xi) \rightarrow\left(Q^{\prime}, \delta^{\prime}, \xi^{\prime}\right)$ be a 
ג-homomorphism with image $I=\{f(q) \mid q \in Q\}$ and with $i: I \rightarrow Q^{\prime}$ the inclusion. Then if $p: Q \rightarrow I$ has $p(q)=f(q)$, there exist zonique $\delta^{\prime \prime}: I X \rightarrow I, \xi^{\prime \prime}: I T \rightarrow I$ such that $p:(X, \delta, \xi) \rightarrow\left(I, \delta^{\prime \prime}, \xi^{\prime \prime}\right)$ and $i:\left(I, \delta^{\prime \prime}, \xi^{\prime \prime}\right) \rightarrow\left(Q^{\prime}, \delta^{\prime}, \xi^{\prime}\right)$ are $\lambda$-homomorphisms. Moreover, given any other diagram of $\lambda$-homomorphisms

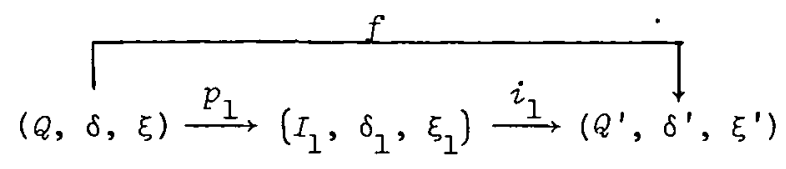

with $p_{1}$ surjective, $i_{1}$ injective, there exists a unique $\lambda$-isomorphism $\Gamma$ as shown below:

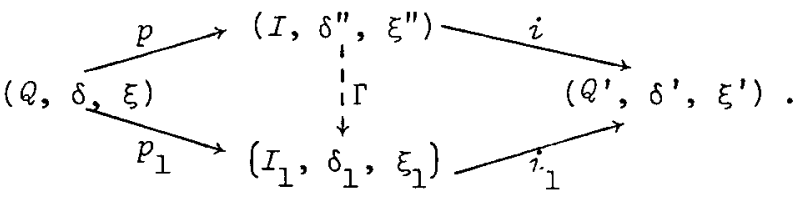

Proof. As $p$ is onto, there exists $d: I \rightarrow Q$ with $p d=i d_{I}$. As $T$ is a functor, $p T d T=i d_{I T}$,

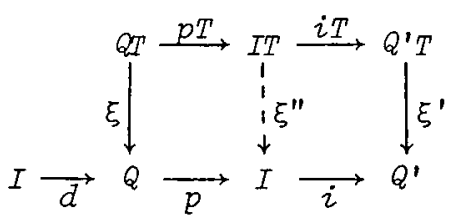

and $p T$ is onto. We leave the remainder of the proof to the reader with the following three hints: $\xi^{\prime \prime}$ as above exists uniquely because $p T$ is onto, $i$ is injective and the perimeter commutes; the two decider axioms and the $\lambda$-law of Definition 1 follow from the principle that to prove $f, g: A \rightarrow I$ are equal it suffices to prove $i \cdot f=i \cdot g ; \Gamma$ is defined by $\Gamma(p(q))=p_{1}(q)$.

With the motivation provided by 17 , Section 1 , we may immediately give the definitions of $\lambda$-machine and implicit $\lambda$-machine:

DEFINITION 5. A $\lambda$-machine is a 7 -tuple $M=(Q, \delta, I, \tau, Y, \theta, \beta)$ where $(Y, \theta)$ is a $T$-decider and

$$
I \stackrel{\tau}{\longrightarrow} Q T, Q X \stackrel{\delta}{\longrightarrow} Q T, Q \stackrel{B}{\longrightarrow} Y
$$


are K-morphisms (the initial state, dynamics, and output map, respectively).

While this definition is independent of which $\lambda$ relates $X$ and $T$, the way such machines "run" - as described in Sections 6 and 7 - is not. A similar definition was presented by Burroni in [7].

DEFINITION 6. An implicit $\lambda$-machine is an 8-tuple $\bar{M}=(\bar{Q}, \bar{\delta}, \bar{\xi}, I, \bar{\tau}, Y, \theta, \bar{\beta})$ where

$(\bar{Q}, \bar{\delta}, \bar{\xi})$ is a $\lambda$-algebra,

$\bar{\tau}: I \rightarrow \bar{Q}$ is a $K$-morphism, and

$\bar{\beta}:(\bar{Q}, \bar{\xi}) \rightarrow(Y, \theta)$ is a T-homomorphism.

Observe that both definitions collapse to the usual one in the deterministic case where $T$ is the identity theory as in Example 2. The difficulties encountered in fuzzy minimal realization theory are a direct consequence of the fact that the algebraic machinery producing unique reachable-observable realizations depends on Definition 6 , whereas what we really want are "states" which require Definition 5. The proof that these two types of machine can simulate each other is given in Section 7 .

Recall that in the classic case, 10-13, Section 1, we passed from a nondeterministic sequential machine (that is, $\lambda$-machine)

$$
\left\{\tau \in 2^{Q} ; \delta: Q \times X_{0} \rightarrow 2^{Q} ; \beta: Q \rightarrow\{0,1\}\right\}
$$

to a deterministic sequential machine which is an implicit $\lambda$-machine

$$
\left\{\tau: I \rightarrow 2^{Q} ; \bar{\delta}=\delta \circ Q \lambda: 2^{Q} \times X_{0} \rightarrow 2^{Q} ; \beta^{\#}: 2^{Q} \rightarrow\{0,1\}\right) \text {. }
$$

We now show that $\left(Q T, \delta^{\#} \cdot Q \lambda=\delta^{\circ} Q \lambda, Q m\right)$ is a $\lambda$-algebra for general $X$, $T, \lambda$, and any $X$-dynamics $(Q, \lambda)$. We know (Theorem 7 , Section 4 ) that we can always pass from an output map $\beta: Q \rightarrow Y$ with a T-decider structure $(Y, \theta)$ on $Y$ to the unique $T$-homomorphism $\beta^{\#}=\theta \cdot \beta T$ which extends $B$.

LEMMA 7. For any $Q X \stackrel{\delta}{\longrightarrow} Q T,\left(Q T, \delta^{\#} \cdot Q \lambda, Q m\right)$ is a $\lambda$-algebra. 
Proof.

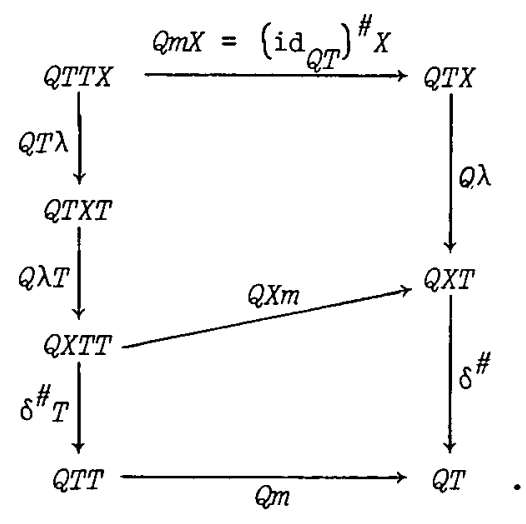

The top piece is an instance of the square of 3.4 with $\alpha=$ id $_{Q T}$ since $Q m=\left(\mathrm{id}_{Q T}\right)^{\#}$ and $(Q \lambda)^{\#}=Q X m \cdot Q \lambda T$.

The bottom piece just says that $\delta^{\#}$ is a $T$-homomorphism.

Our next result generalizes the observation that any sequential machine dynamics $\delta: Q \times X_{0} \rightarrow Q_{0}$ gives rise to a nondeterministic dynamics

$$
2^{Q} \times X_{0} \stackrel{Q \lambda}{\longrightarrow} 2^{Q \times X_{0}} \stackrel{2^{\delta}}{\longrightarrow} 2^{Q}:(p, x) \rightarrow\{\delta(q, x) \mid q \in p\} .
$$

Moreover, this construction lifts dynamorphisms $\beta$ to $\lambda$-homomorphism $\beta^{\#}$ :

THEOREM 8. For each $X$-dynamics $(Q, \delta)$, we have that $(Q T, \delta T \cdot Q \lambda, Q m)$ is a $\lambda$-algebra. Moreover, given any $\lambda$-algebra $(Y, \gamma, \theta)$ and dynomorphism $B:(Q, \delta) \rightarrow(Y, \gamma)$, we have that $B^{\#}=\theta \cdot \beta T$ is a $\lambda$-homomorphism $(Q T, \delta T \cdot Q \lambda, Q m) \rightarrow(Y, \gamma, \theta)$.

Proof. The first claim is just Lemma 7 , with $\delta$ replacing $\delta$. To establish the second statement, we next observe that $\lambda: T X \rightarrow X T$ is a natural transformation; that is,

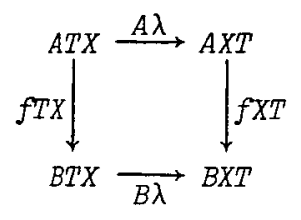


commutes for all $f: A \rightarrow B$. This follows from Definition 4, Section 3, with $\alpha=f^{\Delta}$ since $\left(f^{\Delta} X_{\lambda}\right)^{\#}=(f X)^{\Delta \#}=f X T$. Now use the diagram

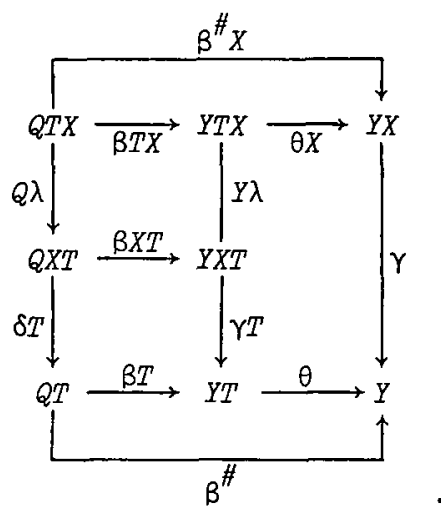

\section{Reachability and observability}

To define reachability for $\lambda$-machines we need to use a free $\lambda$-dynamics; that is, a free $\lambda$-algebra. Given the free $X$-dynamics $\left(I X^{\varrho}, I \mu_{0}\right)$, we may apply the construction of Theorem 8 , Section 5 , to obtain the $\lambda$-algebra $\left(I X^{\varrho} T, I \mu_{0} T \cdot I X^{\varrho} \lambda, I X^{\varrho} m\right)$. We now show that it, too, is free.

THEOREM 1. If $X$ is an input process, then the $\lambda$-algebra $\left(I X^{@} T, I \mu_{0} T \cdot I X^{@} \lambda, I X^{@} m\right)$ is the free $\lambda$-algebra over $I$ with "inclusion of the generators" IX $X^{Q} \cdot I n$.

Proof. We must show that if $(Q, \delta, \xi)$ is any $\lambda$-algebra and $f: I \rightarrow Q$ is any K-morphism,

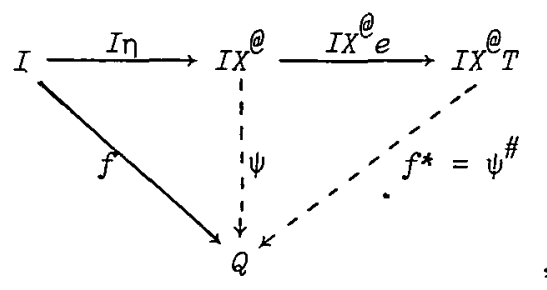

then there exists a unique $\lambda$-homomorphism $f^{*}$ with $f^{*} \cdot I X^{\Theta} e \cdot I n=f$. We 
will show that $f^{*}=\psi^{\#}$ where $\psi$ is the unique dynamorphism from $\left(I X^{Q}, I \mu_{0}\right)$ to $(Q, \delta)$ such that $\psi \cdot I n=f \cdot \psi^{\#}$ is a $\lambda$-homomorphism by Theorem 8, Section 5 .

To prove that $f^{*}$ is unique it suffices to observe that $I X^{\circledR} e$ is a dynamorphism, and this is seen at once from the diagram

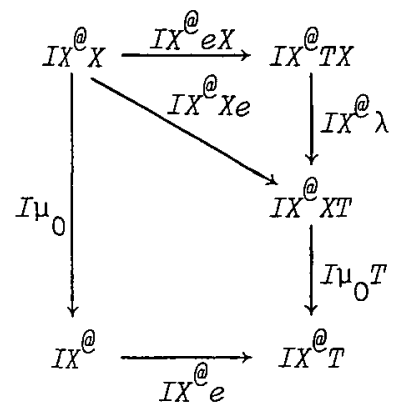

as soon as we note that $e: i d \rightarrow T$ is a natural transformation; that is

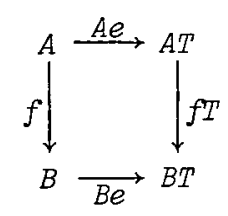

holds for all $f: A \rightarrow B$; for $f T \cdot A e=\left(f^{\Delta}\right)^{\#} \cdot A e=f^{\Delta}=B e \cdot f$.

With this machinery, we may now define the reachability map for a $\lambda$-machine.

DEFINITION 3. The reachability map of the $\lambda$-machine $M$ of Definition 5 , Section 5 , is the $\lambda$-morphism

$$
\tau^{*}:\left(I X^{@} T, I \mu_{0} T \cdot I X^{@} \lambda, I X^{@} m\right) \rightarrow\left(Q T, \delta^{\#} \cdot Q \lambda, Q m\right) .
$$

Notice that the same map arises as the "deterministic" $X_{\lambda}$-reachability map in $K_{T}$ : 


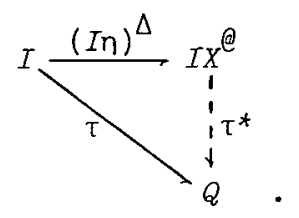

More generally, the reachability map of the implicit $\lambda$-machine $\bar{M}$ of Definition 6 , Section 5 , is the $\lambda$-homomorphism

$$
\tau^{*}:\left(I X^{\Theta} T, I \mu_{0} T \cdot I X^{巴} \lambda, I X^{巴} m\right) \rightarrow(\bar{Q}, \bar{\delta}, \xi) .
$$

We now construct the machinery which lets us build the observability map.

THEOREM 4. If $X$ is an output process then, for each decider $(Y, \theta),\left(Y X_{\varrho}, Y L, \hat{\theta}\right)$ is a $\lambda$-algebra where $\hat{\theta}$ is the conique dynamorphic

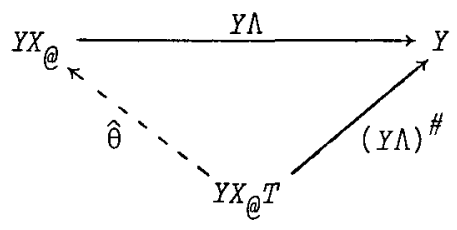

coextension of $(Y \Lambda)^{\#}$, (where $\left(Y X_{\Theta} T, Y L T \cdot Y X_{\Theta^{\lambda}} \lambda, Y X_{\Theta^{m}}\right)$ is a $\lambda$-algebra as in Theorem 8, Section 5). Moreover, for each $\lambda$-algebra $(Q, \delta, \xi)$ and $T$-homomorphism $f:(Q, \xi) \rightarrow(Y, \theta)$, the unique dynamorphic coextension $\psi:(Q, \delta) \rightarrow\left(Y X_{Q}, Y L\right)$ is a $\lambda$-homomorphism.

Proof. We must first show that $\left(Y X_{e}, \hat{\theta}\right)$ is a decider. Since we proved that $Y X_{e^{e}}$ is a dynamorphism in proving Theorem 1 , the diagram

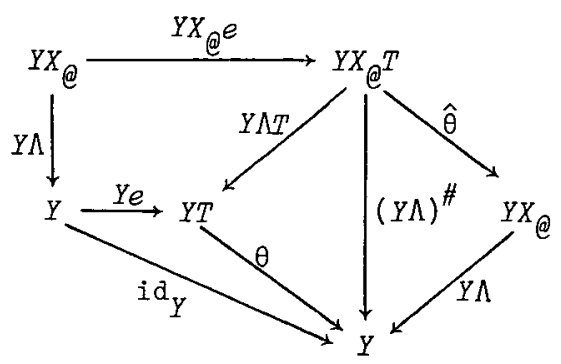


shows that the dynamorphisms $\hat{\theta} \cdot Y X_{e} e$ and $i d_{Y X_{\varrho}}$ are equal followed by $Y \Lambda$, and hence equal. In a similar vein, from the diagram

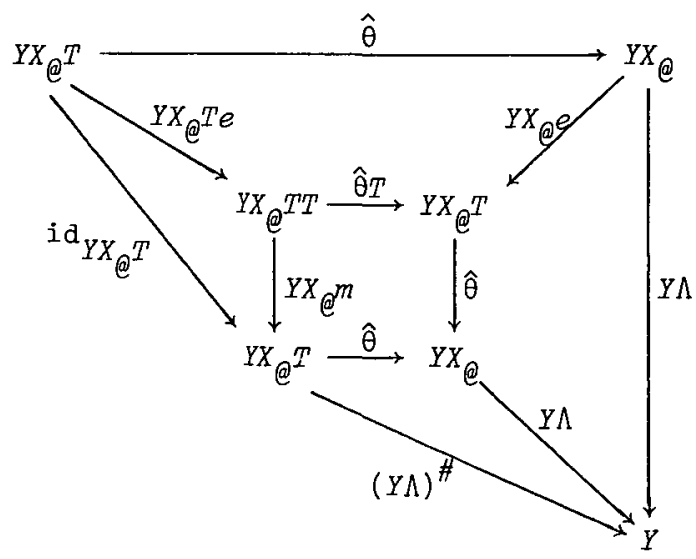

we see first that the $T$-homomorphisms $(Y \Lambda)^{\#} \cdot Y X_{\varrho^{m}}$ and $(Y \Lambda)^{\#} \cdot \hat{\theta} T$ are equal preceded by $Y X_{@} T e$ and hence are equal. We next observe that $\hat{\theta} T$, $Y X^{Q} m$ are dynamorphisms; for use Theorem 8 , Section 5 , with $\beta=(\hat{\theta})^{\Delta}$, id $_{Y X_{Q} T}$ respectively. Finally, the dynamorphisms $\hat{\theta} \cdot \hat{\theta} T, \hat{\theta} \cdot Y X_{Q^{m}}$ are equal followed by $Y \Lambda$, and so are equal. Axiom 1 , Section 5 , is clear since $\hat{\theta}$ is a dynamorphism:

For the second statement, consult the diagram

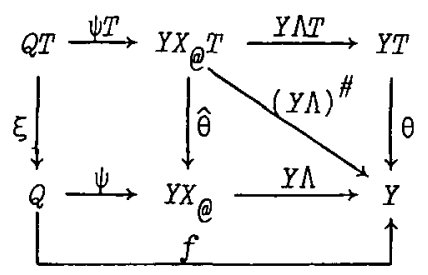

using Theorem 8, Section 5, to see that $\psi T$ is a dynamorphism.

DEFINITION 5. The observability map of the $\lambda$-machine $M$ of Definition 5 , Section 5 , is the $\lambda$-homomorphism

$$
\sigma:\left(Q T, \delta^{\#} \cdot Q \lambda, Q m\right)+\left(Y X_{\varrho}, Y L, \hat{\theta}\right)
$$

which is the unique dynamorphic coextension of the $T$-homomorphism 
$\beta^{\#}:(Q T, Q m)+(Y, \theta)$.

More generally, the observability map of the implicit $\lambda$-machine $\bar{M}$ of Definition 6 , Section 5 , is the $\lambda$-homomorphism

$$
\sigma:(\bar{Q}, \bar{\delta}, \xi) \rightarrow\left(Y X_{\varrho}, Y L, \hat{\theta}\right)
$$

which is the unique dynamorphic coextension of the $T$-homomorphism $\bar{\beta}:(\bar{Q}, \xi) \rightarrow(Y, \theta)$.

The total response map of $M$ (or $\bar{M}$ ) is the $\lambda$-homomorphism

$$
\left(I X^{Q} T, I \mu_{0} T \cdot I X^{Q} \lambda, I X^{Q} m\right) \stackrel{\sigma \cdot \tau^{*}}{\longrightarrow}\left(Y X_{\Theta}, Y L, \hat{\theta}\right) \text {. }
$$

\section{Minimal realization}

In this section we will always assume $X$ is an input process. A morphism of the form $f: I X^{\Theta} \rightarrow Y$ may be the response of either a $\lambda$-machine or an implicit $\lambda$-machine. Indeed, given either machine, we shall see (Theorem 10) that there exists a suitable machine of the other sort so that both have the same response. We shall see that while the familiar algebraic construction of the minimal deterministic realization generalizes easily to implicit $\lambda$-machines (Proposition 4 ), the associated $\lambda$-machine need not have the "minimal number of states".

Let us first dispatch the generalities. In the minimal realization theory for state-behavior machines [4], one factorizes Dyn $(X)$-morphisms

$$
I F \rightarrow Y G \text {, }
$$

where the left and right adjoints $F$ and $G$ of the forgetful functor $U: \operatorname{Dyn}(X) \rightarrow K$ yield $I F=\left(I X^{\complement}, I \mu_{0}\right)$ and $Y G=\left(Y X_{\varrho}, Y L\right)$.

In the present study, our total response maps (Definition 5, Section 6) have the form of $k^{\lambda}$-morphisms

$$
I F \rightarrow Y G,
$$

where it is the left and right adjoints $F$ and $G$ of the forgetful functor $U: K^{\lambda} \rightarrow K$ that yield $I F=\left(I X^{Q} T, I \mu_{0} T \cdot I X^{Q} \lambda, I X^{Q} m\right)$ (by Theorem 1, Section 6) and $G Y=\left(Y X_{\varrho}, Y L, \hat{\theta}\right)$ (by Theorem 4, Section 6). 
In both cases, then, we have a minimal realization theory for A-morphisms

$$
I F \rightarrow Y G,
$$

where $U: A \rightarrow B$ is a functor with left adjoint $F$ and right adjoint $G$. This then suggests a general axiomatic treatment of minimal realization (which is in fact implicit in the work of Bainbridge [5] - see [3, p. 58] for further references).

DEFINITION 1. Let $A$ be a category equipped with a functor $U: A \rightarrow B$. Then an A-machine is $(D, \tau, B)$, where

$\tau: I \rightarrow D U$ is a B-morphism called the input map; and

$B: D U \rightarrow Y$ is a B-morphism called the output map.

A homomorphism of A-machines, $\left(D_{1}, \tau_{1}, \beta_{1}\right) \rightarrow\left(D_{2}, \tau_{2}, \beta_{2}\right)$ with the same $I$ and $Y$ is an A-morphism $\Gamma: D_{1} \rightarrow D_{2}$ such that

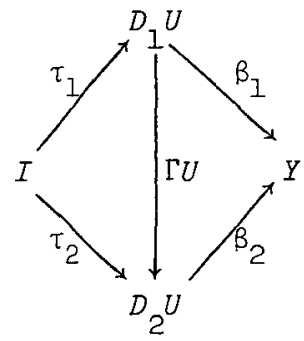

With this definition, such machines form a category $\operatorname{Mach}(A, I, Y)$.

DEFINITION 2. If $U: A+B$ has left adjoint $F$, we define the reachability map of $M=(D, \tau, \beta)$ to be the unique A-morphism $r: I F \rightarrow D$ satisfying

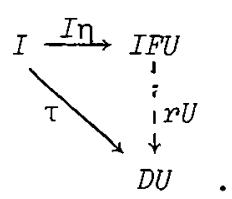

If $U$ has right adjoint $G$, we define the observability map of $M$ to be the unique A-morphism $\sigma: D \rightarrow Y G$ satisfying 


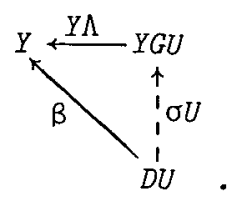

If $U$ has both left and right adjoints, we call $\phi=\sigma \cdot r:$ IF $\rightarrow Y_{G}$ the total response map of $M$. We call $M$ a realization of $\phi$.

Given a factorization system ${ }^{3}(E, M)$ for $B$, we say $M$ is reachable if $r U$ is in $E$, observable if $\sigma U$ is in $M$.

We immediately have the:

AXIOMATIC MINIMAL REALIZATION THEOREM 3. Let $U: A \rightarrow B$ be a functor with left adjoint $F$, and right adjoint $G$. Let $(E, M)$ be a factorization system for $B$ with the property

IF Given any A-morphism $f: A_{1} \rightarrow A_{2}$, and an $E-M$ factorization

$$
A_{1} U \stackrel{P}{\longrightarrow} B \stackrel{\circ}{\longrightarrow} A_{2} U
$$

of $f U$, there exists a unique A-object $A$ and unique A-morphisms $\bar{p}: A_{1} \rightarrow A$ and $\bar{\sigma}: A \rightarrow A_{2}$ such that $\overline{p U}=p$ and $\bar{\sigma} U=\sigma$;

THEN for every A-morphism

$$
\phi: I F \rightarrow Y G
$$

there exists on A-machine $\bar{M}_{\phi}$ which is a reachable and observable realization of $\phi$; moreover $\bar{M}_{\phi}$ is unique up to isomorphism in Mach(A,I, Y).

Proof. We simply use the diagram

\footnotetext{
3 At this level of axiomatic treatment, we do not need an image factorization system - all we require is that each map factorizes into an $E$ followed by an $M$, and that this factorization is unique up to i somorphism.
} 


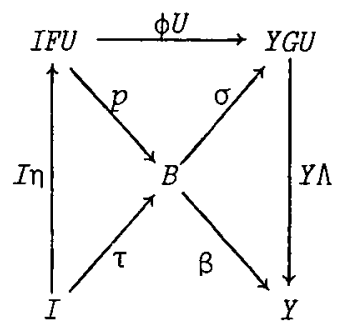

to define $\tau$ and $\beta$ in terms of the (E,M) factorization of $\phi U$. Let then $\bar{p}: I F \rightarrow A$ and $\bar{\sigma}: A \rightarrow Y G$ satisfy $\bar{p} U=p$ and $\bar{\sigma} U=\sigma$. Then $\bar{p}$ and $\bar{\sigma}$ are the reachability and observability maps of $(A, \tau, B)$, which is thus a reachable and observable realization of $\phi$. Uniqueness up to isomorphism is immediate.

Stripped to its essentials like this, minimal realization seems rather dull!

If we specialize all this to $K^{\lambda} \rightarrow K^{\top}$, with $K=S t$, $X=-\times X_{0}:$ Set + Set, $\lambda$ as in Proposition 6, Section $3,(y, \theta)$ a $T$-decider and $I$ a one-element set so that $I X^{\complement}=X_{0}^{*}$, we use Lemma 4 , Section 5 to immediately obtain

PROPOSITION 4. For every $\lambda$-homomorphism $\phi: X_{0}^{*} T \rightarrow Y_{0}^{X^{*}}$, there exists an implicit $\lambda$-machine $\bar{M}_{\phi}$ which is a reachable and observable realization of $\phi ;$ moreover, $\vec{M}_{\phi}$ is unique up to isomorphism.

We now turn to the relationship between $\lambda$-machines and implicit $\lambda$-machines. For this we only require that $X$ be an input process, and so study the response map $f_{M}: I X^{\circledR} \rightarrow Y$ rather than the total response map $\phi_{M}: I X^{\varrho} T \rightarrow Y X_{\varrho}$.

DEFINITION 5 . The response $f_{M}$ of the $\lambda$-machine $M$ of Definition 5 , Section 5 is the response of the deterministic $X$-machine

$$
I \stackrel{\tau}{\longrightarrow} Q T, Q T X \stackrel{Q \lambda}{\longrightarrow} Q X T \stackrel{\delta^{\#}}{\longrightarrow} Q T, Q T \stackrel{B^{\#}}{\longrightarrow} Y ;
$$

that is, $f_{M}$ is the K-morphism 


$$
\begin{gathered}
\text { Fuzzy machines in a category } \\
\text { • } \\
I X^{\Theta} \stackrel{r}{\longrightarrow} Q T \stackrel{\beta^{\#}}{\longrightarrow} Y,
\end{gathered}
$$

where $r$ is the reachability map of (4), Section 1; that is, the unique dynamorphic extension of $\tau$.

Since $\tau^{*} \cdot I X^{Q} e$ is a dynamorphism and $\left(\tau^{*} \cdot I X^{e} e\right) \cdot I n=\tau$, $\tau^{*} \cdot I X^{Q} e=r$. We then have the following relationships (delete $Y \Lambda \cdot \sigma$ if $X$ is not output):

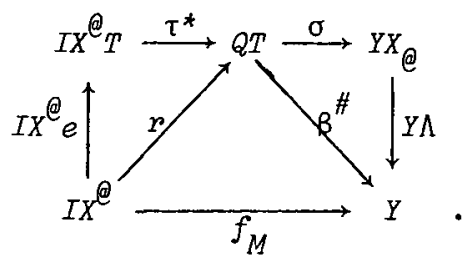

Thus the total response map $\sigma \cdot \tau^{*}$ is the unique $T$-homomorphic extension of the dymamorphic coextension $\sigma \cdot r$ of $f_{M}=\beta^{\#} \cdot r$.

DEFINITION 7. The response $f_{\vec{M}}$ of the implicit $\lambda$-machine of Definition 6 , Section 5 , is the composition

$$
I X^{\varrho} \stackrel{r}{\longrightarrow} \bar{Q} \stackrel{\bar{B}}{\longrightarrow} Y
$$

where $r$ is the unique dynamorphic extension of $\tau$.

It is clear from the definitions that if $M$ is a $\lambda$-machine, then its associated deterministic machine $\left(Q T, \delta^{\#} \cdot Q \lambda, Q m, I, \tau, Y, \beta^{\#}\right)$ is an implicit $\lambda$-machine whose response is $f_{M}$. We now set out to establish an appropriate converse:

DEFINITION 8. If $(\bar{Q}, \bar{\xi})$ is a T-decider, a scoop of $(\bar{Q}, \bar{\xi})$ is a triple $(Q, i, c)$ where

$$
Q \stackrel{i}{\rightarrow} \bar{Q} \stackrel{e}{\longrightarrow} Q T
$$

are such that $i^{\#} \cdot c=i d_{\bar{Q}}$ (where $i^{\#}=\bar{\xi} \cdot i T$ ).

The terminology is due to Ehrig et al. [8, 10.3] and our definition is a variation of theirs. Notice, however, that $(\bar{Q}, i d, \bar{Q} e)$ is always a scoop. 
PROPOSITION 9. Given an implicit $\lambda$-machine $\bar{M}$ then any scoop $(Q, i, c)$ of $(\bar{Q}, \bar{\xi})$ provides the state object $Q$ for a $\lambda$-machine $M$ with $f_{M}=f_{\bar{M}} ; M$ is defined by

$$
\begin{aligned}
& \delta=Q X \stackrel{i X}{\longrightarrow} \bar{Q} X \stackrel{\bar{\delta}}{\longrightarrow} \bar{Q} \stackrel{c}{\longrightarrow} Q T, \\
& \tau=I \stackrel{\bar{\tau}}{\longrightarrow} \bar{Q} \stackrel{c}{\longrightarrow} Q T, \\
& \beta=Q \stackrel{i}{\longrightarrow} \bar{Q} \stackrel{\bar{B}}{\longrightarrow} Y .
\end{aligned}
$$

Proof. We prove that the diagram

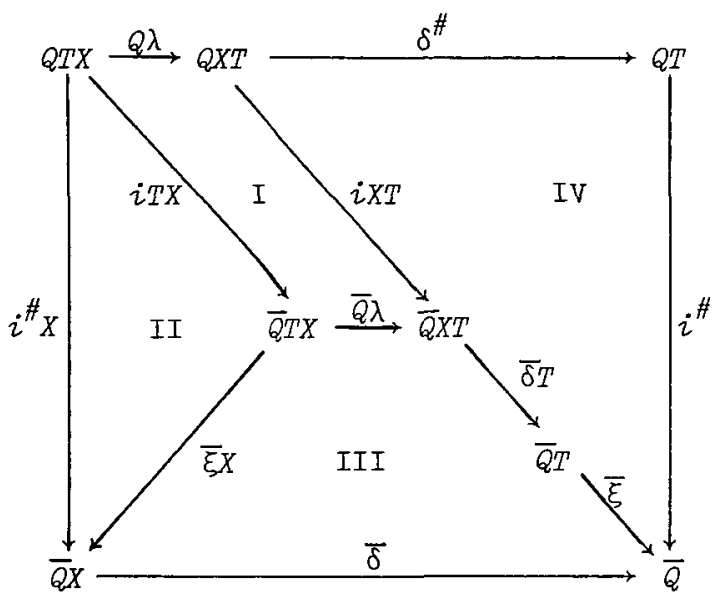

commutes. I commutes since $\lambda$ is a natural transformation. II commutes since $i^{\#}=\bar{\xi} \cdot i T$. III just says that $(\bar{Q}, \bar{\delta}, \bar{\xi})$ is a $\lambda$-algebra. Since $i^{\# \cdot c}=i d \vec{Q}$ by the definition of 'scoop', we have

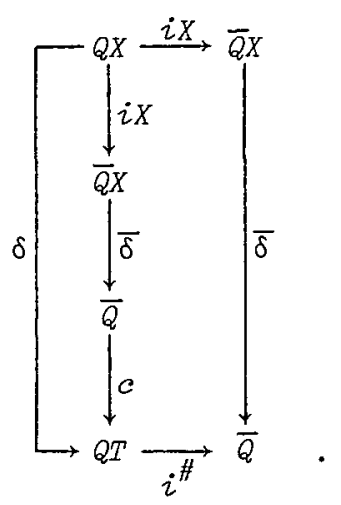


Noting that the two paths of IV yield T-homomorphisms, we recall Theorem 7 , Section 4 , to see that they are equal since, when preceded by QXe , both equal $\bar{\delta} \cdot i X$ :

$$
i^{\#} \cdot \delta^{\#} \cdot Q X e=i^{\#} \cdot \delta=\bar{\delta} \cdot i X
$$

by the last diagram while

$$
\begin{aligned}
\bar{\xi} \cdot \bar{\delta} T \cdot i X T \cdot Q X e & =\bar{\xi} \cdot\left((\bar{\delta} \cdot i X) T^{\Delta} \circ Q X e\right) \\
& =\bar{\xi} \cdot(\bar{\delta} \cdot i X) T^{\Delta} \\
& =\bar{\xi} \cdot Q e \cdot(\bar{\delta} \cdot i X) \\
& =\bar{\delta} \cdot i X .
\end{aligned}
$$

Since $\bar{B}$ is a T-homomorphism we also have

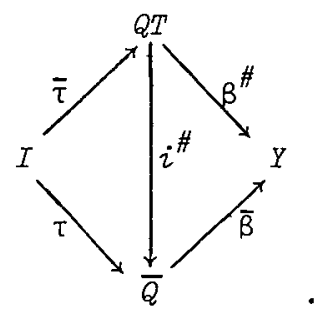

Thus $i^{\#}:\left(Q T, \delta^{\#} \cdot Q \lambda, I, \tau, Y, \beta^{\#}\right) \rightarrow(\bar{Q}, \bar{\delta}, I, \bar{\tau}, Y, \bar{\beta})$ is a homomorphism of $X$-machines and, in particular, $f_{M}=f_{\bar{M}}$.

We immediately have

THEOREM 10. Let there be given $f: I X^{\circledR} \rightarrow y$, a T-decider structure $(Y, \theta)$ and an object $Q$ of $K$. Then a $\lambda$-machine $M$ with state object $Q$ and response $f_{M}$ equal to $f$ exists iff there exists an implicit $\lambda$-machine $M$ with $f_{\bar{M}}=f$ and whose decider $(\bar{Q}, \bar{\xi})$ admits a scoop $\left(Q^{\prime}, i, c\right)$ with $Q$ and $Q^{\prime}$ isomorphic in $K$.

Proof. $\left(Q, Q e, i d_{Q T}\right)$ is a scoop of $(Q T, Q m)$.

In the next section, we characterize scoops in terms of extremal elements.

\section{Examples}

We now relate the scoops of Definition 8 , Section 7 to extremal 
elements; and then give examples of realization by nondeterministic automata. Throughout this section we restrict $K$ to be Set .

If $(\bar{Q}, \xi)$ is a T-decider and $Q$ is a subset of $\bar{Q}$, let $\langle\dot{Q}\rangle$ denote the image of $i^{\#}$ in $Q$ where $i: Q \rightarrow \bar{Q}$ is the inclusion function. In the context of Examples 2, 3, 4 of Section $4,\langle Q\rangle$ is, respectively, the submonoid generated by $Q$, the union of all $M$-orbits intersecting $Q$, and the set of all 'convex combinations' of elements in $Q$. For $T$ as in (5), Section $2,\langle Q\rangle$ is the set of all suprema of elements of $Q$.

$Q$ generates $(\bar{Q}, \xi)$ if $\langle Q\rangle=\bar{Q}$; that is, $(Q, i, c)$ is a scoop for some $c \cdot \bar{q}$ in $\bar{Q}$ is an isolated element of $(\bar{Q}, \xi)$ if $q \mid(\bar{Q} \backslash\{q\}) .(\bar{Q}, \xi)$ is extremal if $(\bar{Q}, \xi)$ is generated by its isolated elements (in which case the isolated elements are contained in every set of generators and so constitute the unique minimal set of generators), and $T$ is extremal if every finitely-generated T-decider is extremal.

The $T$ of Example 10, Section 2, is not extremal (the 3-element group is generated by either of its non-units) even though free monoids are.

Isolated elements of convex sets are the well-known extreme points and thus finitely-generated convex sets are extremal. We do not know if all finitely-generated stochastic deciders are extremal, but this is true for all doubly-generated stochastic deciders (classified in [14, 4.3, Exercise 1]) which, incidentally, would appear to be an intriguing generalization of Zadeh's unit interval as a universe for "assigning weights to two truth values consistent with the laws of probability theory".

With respect to $T$ as in (5), Section 2, an extremal element of a complete semi-lattice is more conventionally called a join-irreducible. This $T$ is extremal:

PROPOSITION 1. Every finite (equals finitely-generated complete) semilatice $\bar{Q}$ is extremal.

Proof. Let $Q_{1}$ be the set of minimal elements of $\bar{Q} \backslash\{0\}$ ( 0 denotes the empty supremum; that is, the least element) and let $Q_{n+1}$ be the set of minimal elements of $\vec{Q} \backslash\left\langle Q_{1} \cup \ldots \cup Q_{n}\right\rangle$ (where $\langle A\rangle$ denotes the set of 
suprema of elements of $A)$, continuing until $\vec{Q}=\left(Q_{1} \cup \ldots \cup Q_{m}\right\rangle$. It suffices to prove that each $Q_{n}$ consists of join-irreducibles. This is clear for $n=1$. Suppose $q \in Q_{n+1}$ and $q=q_{1} \vee \ldots \vee q_{k}$ with no $q_{i}=q$. Since $q \notin\left\langle Q_{1} \cup \ldots \cup Q_{n}\right\rangle, k \neq 0$. Since each $q_{i}<q$, we must have $q_{i} \in\left(Q_{1} \cup \ldots \cup Q_{n}\right\rangle$, the desired contradiction.

Recalling the conventions stated just before Definition 7, Section 7, we have

DEFINITION 2. A finite-state minimal realization of $f: X_{0}^{*} \rightarrow Y$ is an implicit $\lambda$-machine $\bar{M}=(\bar{Q}, \bar{\delta}, \bar{\xi}, \bar{\tau}, \bar{\beta})$ and a scoop $(Q, i, c)$ of $(\bar{Q}, \bar{\xi})$ satisfying

(i) $f_{\bar{M}}=f$,

(ii) $Q$ is finite,

(iii) subject to (i), (ii) the number of elements of $Q$ is as small as possible.

In this context, Theorem 10, Section 7 asserts

THEOREM 3. To find a nondeterministic minimal realization of $f$ it is necessary and sufficient to find an implicit nondeterministic realization of $f$ having fewest possible join-irreducibles.

In the deterministic case, $T=i d, Q=\bar{Q}$ is the only scoop and the notion is the usual one and coincides with the unique reachable and observable realization. The following example shows, however, that 'minimal' need not even imply 'reachable'.

EXAMPLE 4. We now present an example due to Ehrig et al. [8, 10.6] of a response whose nondeterministic minimal realization has fewer joinirreducibles than the realization of Proposition 4, Section 7 . Let $X_{0}=\{x\}$ have one element, let $Y$ be the semilattice of subsets of $\{a, b, c, d\}$ and let $f: X_{0}^{*} \rightarrow Y$ be the sequence

$$
f=a / b / c / d / / a b / a c / a d / /
$$

(that is, $f\left(x^{0}\right)=a, f\left(x^{4}\right)=f\left(x^{7}\right)=\{a, b\} ; / /$ denotes cycling). 
As is well known, the state space $Q_{f}$ of the deterministic minimal realization of $f$ may be constructed as the closure under the left-shift of $f$ in $y^{X_{0}^{*}}$. Writing fw for $f L_{w}$, we readily compute

$$
\begin{aligned}
f x & =b / c / d / / a b / a c / a d / /, \\
f x^{2} & =c / d / / a b / a c / a d / /, \\
f x^{3} & =d / / a b / a c / a d / /, \\
f x^{4+3 k} & =/ / a b / a c / a d / /, \\
f x^{5+3 k} & =/ / a c / a d / a b / / \\
f x^{6+3 k} & =/ / a d / a b / a c / /
\end{aligned}
$$

so that $Q_{f}$ has the seven states: $Q_{f}=\left\{f, \ldots, f x^{6}\right\}$. Set $\bar{Q}$ to be the subset of $Y^{X_{0}^{*}}$ of all suprema (that is, pointwise unions) of elements of $Q_{f}$. Then $\bar{Q}$ is closed under left shift and is easily seen to be an FSO . Moreover, $\bar{Q}$ becomes an implicit nondeterministic realization of $f$ with $\vec{\tau}=f$ and $\vec{\beta}(g)=g(\Lambda)$ where $\Lambda$ is the empty word in $X_{0}^{*}$. It is clear from the earlier discussion of isolated elements that the joinirreducibles of $\bar{Q}$ are just those elements of $Q_{f}$ which cannot be expressed as suprema of other elements of $Q_{f}$. In this case, the only such relation is $f x^{6}=f \vee f x^{3}$, so that $\bar{Q}$ has six join-irreducibles. The state-graph of the corresponding nondeterministic sequential machine as in the proof of Proposition 9, Section 7 (notice that we only use the values of $c$ on elements of form $\bar{\tau}$ or $\bar{\delta}(q, x)$ and that on these values $c$ is unique in this particular example) is

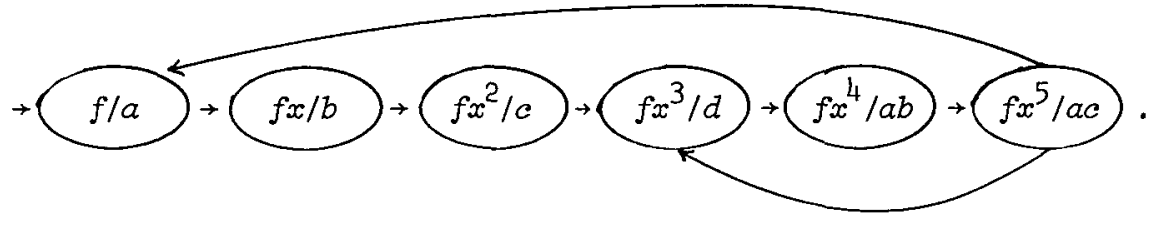


Let $\tilde{a} \in Y^{X_{0}^{*}}$ be constantly $a$. Then $\bar{R}=\bar{Q} \cup\{\bar{a}\}$ is an FSO containing $\bar{Q}$ as a sub-RSO. However, $\bar{R}$ has only the five joinirreducibles $R=\left\{f, f x, f x^{2}, f x^{3}, \tilde{a}\right\}$ owing to the relationships $f x \vee \tilde{a}=f x^{4}, f x^{2} \vee \tilde{a}=f x^{5}, f x^{6}=f \vee f x^{3} \vee \tilde{a}$. The corresponding nondeterministic sequential machine is

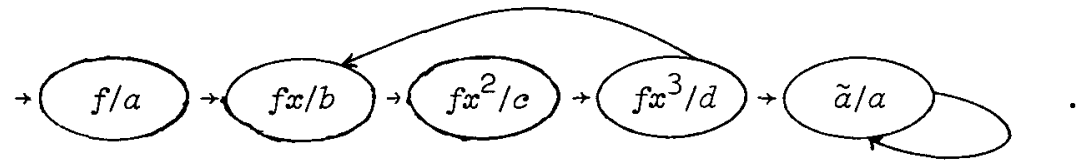

It is obvious that no nondeterministic realization of $f$ can have only four states, so this realization is minimal. The image of the reachability map $\tau^{*}: 2^{\{x\}^{*}} \rightarrow \bar{R}$ (as in Theorem 3, Section 7) does not contain $\tilde{a}$.

EXAMPLE 5. We present a simple example of a response possessing two minimal realizations corresponding to non-isomorphic implicit realizations (only one is reachable and observable). Let $X_{0}=\{x\}$ have one element, let $Y$ be the semilattice of subsets of $\{a, b\}$ and let $f: X_{0}^{*} \rightarrow Y$ be the sequence

$$
q_{0}=f: a / b / a / / a b / /
$$

(for notations see Example 4). Then

$$
\begin{aligned}
& q_{1}=f x=b / a / / a b / /, \\
& q_{2}=f x^{2}=a / / a b / /, \\
& q_{3}=f x^{3+k}=/ / a b / / .
\end{aligned}
$$

Then $q_{3}=q_{2} \vee q_{1}=q_{0} \vee q_{1}$ giving rise to two possible scoops and the following two $\lambda$-machines
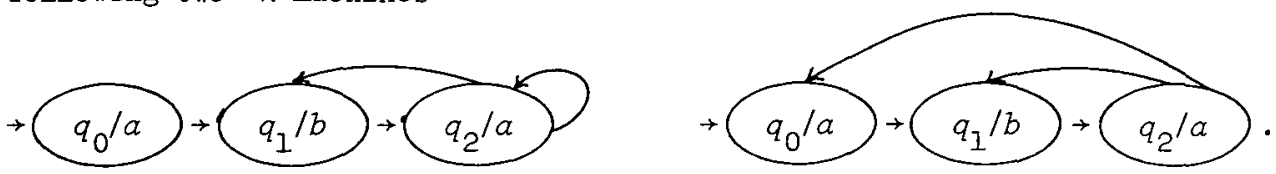

The $\lambda$-algebra structure in both cases is 


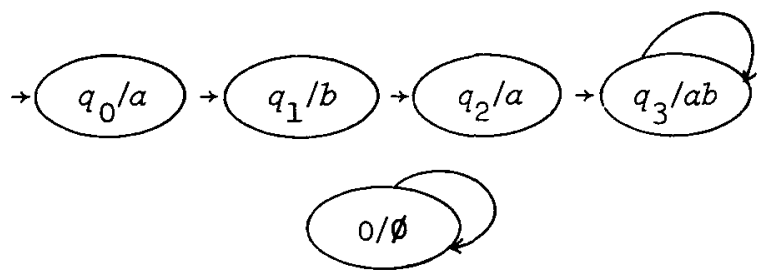

Dynamic Structure

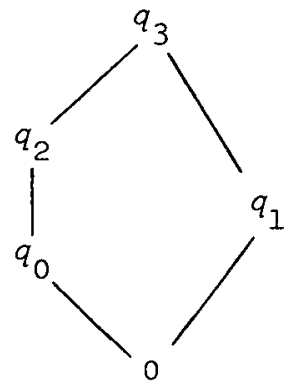

Semilattice Structure

It is easy to check that $f$ cannot be realized using only two states, so this implicit realization is minimal. Now consider the following threestate $\lambda$-machine which realizes $f$.

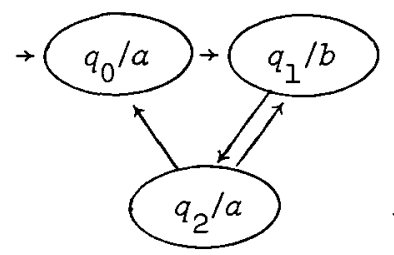

The corresponding implicit $\lambda$-machine is shown below:
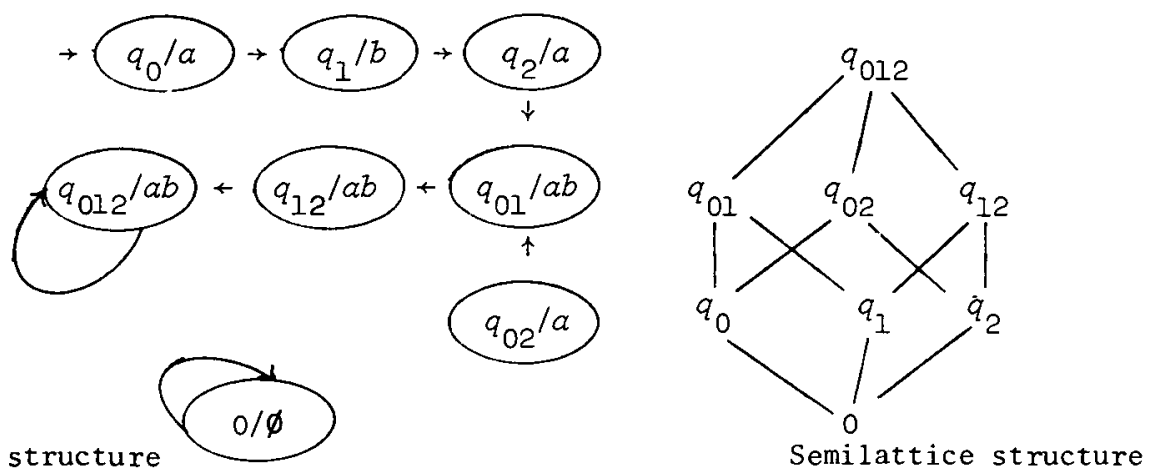

Both implicit realizations are reachable but only the first is observable. 


\section{References}

[1] Michael A. Arbib and Ernest G. Manes, "Machines in a category: an expository introduction", SIAM Rev. 16 (1974), 163-192.

[2] Michael A. Arbib, Ernest G. Manes, Arrows, structures, and functors. The categorical imperative (Academic Press, New York, San Francisco, London, 1975).

[3] M.A. Arbib, E.G. Manes, "A categorist's view of automata and systems", Category theory applied to computation and control, 51-64 (Lecture Notes in Computer Science, 25. Springer-Verlag, Berlin, Heidelberg, New York, 1975).

[4] Michael A. Arbib, Ernest G. Manes, "Adjoint machines, state-behavior machines, and duality", J. Pure Appl. Algebra (to appear).

[5] Edwin Stewart Bainbridge, "A unified minimal realization theory with duality ( $\mathrm{PhD}$ thesis, University of Michigan, Michigan, 1972).

[6] Jon Beck, "Distributive laws", Seminar on triples and categorical homology theory, 119-140 (Lecture Notes in Mathematics, 80. Springer-Verlag, Berlin, Heidelberg, New York, 1969).

[7] Élisabeth Burroni, "Algèbres relatives à une loi distributive", C.R. Acad. Sci. Paris Sér. A 276 (1973), 443-446.

[8] H. Ehrig, K.-D. Kiermeier, H.-J. Kreowski and W. Kühnel, Universal theory of automata. A categorical approach (Teubner, Stuttgart, 1974 ).

[9] J.A. Goguen, "L-fuzzy sets", J. Math. Anal. Appl. 18 (1967), 145-I74.

[10] J.A. Goguen, "Minimal realization of machines in closed categories", Buzz. Amer. Math. Soc. 78 (1972), 777-783.

[11] J.A. Goguen, "Concept representation in natural and artificial languages: axioms, extensions and applications for fuzzy sets", Internat. J. Man-Machine Studies 6 (1974), 513-561.

[12] H. Kleisli, "Every standard construction is induced by a pair of adjoint functors", Proc. Amer. Math. Soc. 16 (1965), 544-546. 
[13] S. Mac Lane, Categories for the working mathematician (Graduate Texts in Mathematics, 5. Springer-Verlag, New York, Heidelberg, Berlin, 1971).

[14] Ernest G. Manes, Algebraic theories (Graduate Texts in Mathematics. Springer-Verlag, New York, Heidelberg, Berlin, to appear).

[15] M.O. Rabin, D. Scott, "Finite automata and their decision problems", IBM J. Res. Develop. 3 (1959), 114-125.

[16] George N. Raney, "Sequential functions", J. Assoc. Comput. Mach. 5 (1958), $177-180$.

[17] M.P. Schützenberger, "On a theorem of R. Jungen", Proc. Amer. Math. Soc. 13 (1962), 885-890.

[18] L.A. Zadeh, "Fuzzy sets", Inform. Control 8 (1965), 338-353.

Department of Computer and Information Science, University of Massachusetts,

Amherst,

Massachusetts,

USA;

Department of Mathematics,

University of Massachusetts,

Amherst,

Massachusetts,

USA. 